\title{
HOLOMORPHIC SUBMERSIONS FROM STEIN MANIFOLDS
}

\author{
by Franc FORSTNERIČ
}

\section{Introduction.}

Recall that a Stein manifold is a complex manifold biholomorphic to a closed complex submanifold of a complex Euclidean space $\mathbb{C}^{N}$ [GR, p. 226]. A holomorphic map $f: X \rightarrow Y$ whose differential $d f_{x}: T_{x} X \rightarrow T_{f(x)} Y$ is surjective for every $x \in X$ is said to be a submersion of $X$ to $Y$. The following was proved in [F1, Theorem II]; for $n=q=1$ see also [GN]:

A Stein manifold $X$ admits a holomorphic submersion to $\mathbb{C}^{q}$ for some $q<\operatorname{dim} X$ if and only if its tangent bundle $T X$ admits a surjective complex vector bundle map onto the trivial rank $q$ bundle $\tau_{X}^{q}=X \times \mathbb{C}^{q}$.

The necessity of the latter condition is clear since the tangent map of a submersion $X \rightarrow \mathbb{C}^{q}$ induces a surjective vector bundle map $T X \rightarrow \tau_{X}^{q}$. The corresponding problem for $n=q>1$ remains open [F1].

In this paper we consider the analogous problem with the target space $\mathbb{C}^{q}$ replaced by a more general complex manifold $Y$. The following result in the smooth category was proved by A. Phillips [P] and M. Gromov [Gr1, Gr3]:

$A$ continuous map $f_{0}: X \rightarrow Y$ from a smooth open manifold $X$ to a smooth manifold $Y$ is homotopic to a submersion $f_{1}: X \rightarrow Y$ if and only if there exists a surjective vector bundle map $\iota_{0}: T X \rightarrow f_{0}^{*} T Y$ over $X$, i.e., the pull-back $f_{0}^{*} T Y$ is a quotient bundle of $T X$. The regular homotopy classes of submersions $X \rightarrow Y$ are classified by the homotopy classes of their tangent maps.

In the holomorphic category the topological condition on the existence of $\iota_{0}$ does not suffice. For example, a Kobayashi-hyperbolic manifold $Y$ admits no nonconstant holomorphic images of $\mathbb{C}^{n}$ but the topological condition trivially holds for the constant map $\mathbb{C}^{n} \rightarrow y_{0} \in Y$. This suggests that we restrict the attention to manifolds which admit 'sufficiently many' holomorphic submersions $\mathbb{C}^{n} \rightarrow Y$ for a given $n \geq \operatorname{dim} Y$. It turns out that a suitably precise form of this condition suffices for a complete analogue of the Gromov-Phillips theorem, thus justifying the following heuristic principle (a form of the Oka principle): 
If a complex manifold $Y$ admits sufficiently many holomorphic submersions $\mathbb{C}^{n} \rightarrow Y$ for a given integer $n \geq \operatorname{dim} Y$ then the existence of holomorphic submersions $X \rightarrow Y$ from $n$-dimensional Stein manifolds $X$ reduces to a purely topological problem.

We say that a complex manifold $Y$ satisfies Property $S_{n}$ for some integer $n \geq \operatorname{dim} Y$ if any holomorphic submersion $O \rightarrow Y$ from a compact convex set $O \subset \mathbb{C}^{n}$ of a certain special type can be approximated uniformly on $O$ by holomorphic submersions $\mathbb{C}^{n} \rightarrow Y$ (Definition 1 in Sect. 2). The following is a special case of our main result, Theorem 2.1:

THEOREM. Assume that $X$ is an $n$-dimensional Stein manifold and $Y$ is a complex manifold satisfying Property $S_{n}$. A continuous map $f: X \rightarrow Y$ is homotopic to a holomorphic submersion of $X$ to $Y$ if and only if there exists a surjective complex vector bundle map $\iota: T X \rightarrow f^{*} T Y$.

Furthermore, Property $S_{n}$ is both necessary and sufficient for a stronger version of the above theorem with approximation on compact $\mathcal{O}(X)$-convex subsets of the source manifold $X$; Theorem 2.1 (b). If $Y$ also satisfies the analogous Property $H S_{n}$ concerning the approximation of homotopies of holomorphic submersions $O \rightarrow Y$ by homotopies of submersions $\mathbb{C}^{n} \rightarrow Y$ then for any $n$-dimensional Stein manifold $X$ the regular homotopy classes of holomorphic submersions $X \rightarrow Y$ are classified by the homotopy classes of their tangent maps (Corollary 2.4).

If $n=\operatorname{dim} X \geq 2 \operatorname{dim} Y-1$ then $f^{*} T Y$ is always a quotient of $T X$ by topological reasons and hence every continuous map $X \rightarrow Y$ is homotopic to a holomorphic submersion (Corollary 2.3).

One cannot use Property $S_{n}$ directly since a general submersion $X \rightarrow Y$ does not factor as $X \rightarrow \mathbb{C}^{n} \rightarrow Y$. Instead such decompositions are used on small subsets of $X$ and the resulting local submersions of $X$ to $Y$ are pieced together into a global submersion by the analytic tools developed in [F1] and in this paper.

Properties $S_{n}$ and $H S_{n}$ are equivalent to apparently weaker conditions on uniform approximability of submersions $O \rightarrow Y$ on special compact convex sets $O \subset \mathbb{C}^{n}$ by submersions $\mathbb{C}^{n} \backslash A \rightarrow Y$, where $A \subset \mathbb{C}^{n}$ is an algebraic subvariety of codimension at least two which does not intersect $O$ (this relies upon the theory of holomorphic automorphisms of $\mathbb{C}^{n}$ ). In Sect. 5 we establish Properties $S_{n}$ and $H S_{n}$ when $n>\operatorname{dim} Y=q$ (or $n=q=1$ ) and $Y$ is any of the following: $\mathbb{C}^{q}, \mathbb{C P}^{q}$, a complex Grassmanian, a Zariski open set with thin complement (containing no hypersurfaces) in any of the above, or a holomorphic quotient of any of the above (this class contains all complex tori and Hopf manifolds).

For Riemann surfaces we obtain a complete answer by proving that the following are equivalent (Corollary 2.8):

(a) $Y$ is one of the Riemann surfaces $\mathbb{C P}^{1}, \mathbb{C}, \mathbb{C}^{*}=\mathbb{C} \backslash\{0\}$, or a complex torus (the quotient $\mathbb{C} / \Gamma$ by a rank two lattice $\Gamma \subset \mathbb{C}$ ); 
(b) any continuous map from a Stein manifold $X$ to $Y$ is homotopic to a holomorphic submersion.

The Riemann surfaces listed in (a) are precisely those which are not Kobayashi hyperbolic. When $Y$ is $\mathbb{C}^{*}$ or a torus our result is new even when $X$ is an open Riemann surface.

The heuristic principle behind our main result is reminiscent of Gromov's extension of the Oka-Grauert principle [Gr4]: The existence of many dominating holomorphic maps $\mathbb{C}^{n} \rightarrow Y$ implies the existence of many holomorphic maps $X \rightarrow Y$ from any Stein manifold $X$. A dominating map $s: \mathbb{C}^{n} \rightarrow Y$ based at a point $y \in Y$ is one for which $s(0)=y$ and $d s_{0}: T_{0} \mathbb{C}^{n} \rightarrow T_{y} Y$ is surjective (i.e., $s$ is a submersion near $0 \in \mathbb{C}^{n}$ ). A dominating spray on $Y$ is a family of such maps depending holomorphically on $y \in Y$ (its domain is a holomorphic vector bundle over $Y$ ). Comparing with Property $S_{n}$ we see that the two conditions have similar flavour. For the Oka-Grauert-Gromov theory we refer to [G3, Gr4, HL2, FP1, FP2, FP3, F3, W].

An important ingredient in our construction of submersions is a holomorphic approximation theorem on certain handlebodies in arbitrary complex manifolds; Theorem 3.2 in Sect. 3. This result, together with a geometric lemma from [F1], gives an approximate extension of the submersion across a critical level of a strongly plurisubharmonic exhaustion function in the source manifold. In Sect. 6 we use the same method to give a simple proof of the OkaGrauert principle for sections of holomorphic fiber bundles over Stein manifolds whose fiber admits a finite dominating family of sprays.

\section{The main results.}

We denote by $\mathcal{O}(X)$ the algebra of all holomorphic functions on a complex manifold $X$. A function (or map) is holomorphic on a closed subset $K$ in $X$ if it is holomorphic in some open neighborhood of $K$; the set of all such functions (with the usual identification of functions which agree in a neighborhood of $K$ ) will be denoted $\mathcal{O}(K)$. Any statement concerning a holomorphic map on a closed set should be understood in the sense that it holds in some open neighborhood; for homotopies of maps the neighborhood is the same for all maps in the homotopy. A compact set $K \subset X$ is $\mathcal{O}(X)$-convex if for every $p \in X \backslash K$ there exists $f \in \mathcal{O}(X)$ such that $|f(p)|>\sup _{K}|f|$.

A homotopy of holomorphic submersions $X \rightarrow Y$ is a family of holomorphic submersions $f_{t}: X \rightarrow Y(t \in[0,1])$ depending continuously on $t$. It follows that the tangent maps $T f_{t}: T X \rightarrow T Y$ are also continuous in $t$.

Let $z=\left(z_{1}, \ldots, z_{n}\right)$ be the coordinates on $\mathbb{C}^{n}$, with $z_{j}=x_{j}+i y_{j}$. Set

$$
Q=\left\{z \in \mathbb{C}^{n}:\left|x_{j}\right| \leq 1,\left|y_{j}\right| \leq 1, j=1, \ldots, n\right\} .
$$

A special convex set in $\mathbb{C}^{n}$ is a compact convex subset of the form

$$
O=\left\{z \in Q: y_{n} \leq h\left(z_{1}, \ldots, z_{n-1}, x_{n}\right)\right\},
$$

where $h$ is a smooth (weakly) concave function with values in $(-1,1)$. 
DEFINITION 1. Let $d$ be a distance function induced by a smooth Riemannian metric on complex manifold $Y$.

(a) $Y$ satisfies Property $S_{n}$ if for any holomorphic submersion $f: O \rightarrow Y$ on a special convex set $O \subset \mathbb{C}^{n}$ and any $\epsilon>0$ there is a holomorphic submersions $\widetilde{f}: Q \rightarrow Y$ satisfying $\sup _{x \in O} d(f(x), \widetilde{f}(x))<\epsilon$.

(b) $Y$ satisfies Property $H S_{n}$ if for any homotopy of holomorphic submersions $f_{t}: O \rightarrow Y(t \in[0,1])$ such that $f_{0}$ and $f_{1}$ extend to holomorphic submersions $Q \rightarrow Y$ there exists for any $\epsilon>0$ a homotopy of holomorphic submersions $\widetilde{f}_{t}: Q \rightarrow Y(t \in[0,1])$ satisfying $\widetilde{f}_{0}=f_{0}, \widetilde{f}_{1}=f_{1}$, and $\sup _{x \in O, t \in[0,1]} d\left(f_{t}(x), \widetilde{f}_{t}(x)\right)<\epsilon$.

An obvious induction shows that Property $S_{n}$ of $Y$ implies the following: Any holomorphic submersion $f: O \rightarrow Y$ on a special convex set $O \subset \mathbb{C}^{n}$ can be approximated uniformly on $O$ by holomorphic submersions $\mathbb{C}^{n} \rightarrow Y$. The analogous remark holds for Property $H S_{n}$.

Let $X$ and $Y$ be complex manifolds. Denote by $\mathcal{S}(X, Y)$ the set of all pairs $(f, \iota)$ where $f: X \rightarrow Y$ is a continuous map and $\iota: T X \rightarrow T Y$ is a fiberwise surjective complex vector bundle map making the following diagram commute:

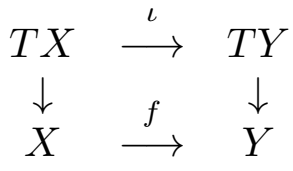

Figure 1: The set $\mathcal{S}(X, Y)$

Such $\iota$ is the composition of a surjective complex vector bundle map $T X \rightarrow$ $f^{*} T Y$ with the natural map $f^{*} T Y \rightarrow T Y$. Let $\mathcal{S}_{\text {holo }}(X, Y)$ consist of all pairs $(f, T f) \in \mathcal{S}(X, Y)$ where $f: X \rightarrow Y$ is a holomorphic submersion and $T f$ its tangent map. We equip $\mathcal{S}(X, Y)$ with the compact-open topology.

The following is our main theorem.

THEOREM 2.1. Assume that $X$ is a Stein manifold of dimension $n$ and $Y$ is a complex manifold satisfying Property $S_{n}$.

(a) (Existence of submersions) Every $\left(f_{0}, \iota_{0}\right) \in \mathcal{S}(X, Y)$ can be connected by a path $\left\{\left(f_{t}, \iota_{t}\right)\right\}_{t \in[0,1]} \subset \mathcal{S}(X, Y)$ to some $\left(f_{1}, T f_{1}\right) \in \mathcal{S}_{\text {holo }}(X, Y)$.

(b) (Approximation) If $K \subset X$ is a compact $\mathcal{O}(X)$-convex subset, $\left.f_{0}\right|_{K}$ is a holomorphic submersion, and $\left.\iota_{0}\right|_{K}=\left.T f_{0}\right|_{K}$ then the path $\left(f_{t}, \iota_{t}\right)$ in (a)can be chosen such that for every $t \in[0,1],\left.f_{t}\right|_{K}$ is a holomorphic submersion uniformly close to $\left.f_{0}\right|_{K}$ and $\left.\iota_{t}\right|_{K}=\left.T f_{t}\right|_{K}$.

(c) (Regular homotopies of submersions) If $Y$ satisfies Property $H S_{n}$ then any path $\xi_{t}=\left(f_{t}, \iota_{t}\right) \in \mathcal{S}(X, Y)(t \in[0,1])$ with $\xi_{0}, \xi_{1} \in \mathcal{S}_{\text {holo }}(X, Y)$ can be deformed with fixed ends to a path in $\mathcal{S}_{\text {holo }}(X, Y)$.

Theorem 2.1 is proved in Section 4. In the special case $Y=\mathbb{C}^{q}$, with $n>q$ or $n=q=1$, parts (a) and (b) were proved in [F1] (for $n=q=1$ see also 
[GN]), but part (c) is new even in this case. The relevant Property $H S_{n}$ holds for $\mathbb{C}^{q}$ whenever $n>q$ (Proposition 2.5 below).

Remarks. 1. If the conclusion of part (b) in Theorem 2.1 holds for a given complex manifold $Y$, with $K$ any compact convex set in $X=\mathbb{C}^{n}$, then (by definition) $Y$ satisfies Property $S_{n}$. Hence both the topological condition (the existence of $\iota$ ) and the analytic condition (Property $S_{n}$ of $Y$ ) are necessary for parts (a) and (b) in Theorem 2.1.

2. Clearly $H S_{n} \Rightarrow S_{n}$. We don't know whether the converse always holds, but in all examples for which we prove $S_{n}$ we also prove $H S_{n}$.

Part (a) of Theorem 2.1 implies the following.

COROLLARY 2.2. If $X$ is an $n$-dimensional Stein manifold and $Y$ satisfies Property $S_{n}$ then for any $(f, \iota) \in \mathcal{S}(X, Y)$ there is a nonsingular holomorphic foliation of $X$ whose normal bundle is isomorphic to $f^{*} T Y$ (as a topological complex vector bundle over $X$ ).

Such a foliation is given by the level sets of a submersion $X \rightarrow Y$ furnished by Theorem 2.1 (a). The corresponding result for foliations with trivial normal bundle (and $Y=\mathbb{C}^{q}$ ) was obtained in [F1].

If $\operatorname{dim} X \geq 2 \operatorname{dim} Y-1$ then every map $f: X \rightarrow Y$ can be covered by a map $\iota: T X \rightarrow T Y$ such that $(f, \iota) \in \mathcal{S}(X, Y)$. This follows by standard topological methods from the fact that an $n$-dimensional Stein manifold is homotopic to an $n$-dimensional CW-complex; it will also be clear from our proof of Theorem 2.1. Hence Theorem 2.1 implies

COROLLARY 2.3. If $Y$ satisfies Property $S_{n}$ for some $n \geq 2 \operatorname{dim} Y-1$ then any map $f: X \rightarrow Y$ from an $n$-dimensional Stein manifold $X$ is homotopic to a holomorphic submersion of $X$ to $Y$.

Combining parts (a) and (c) in Theorem 2.1 we also obtain

COROLLARY 2.4. If a complex manifold $Y$ satisfies Property $H S_{n}$ then for every $n$-dimensional Stein manifold $X$ the natural inclusion

$$
\mathcal{S}_{\text {holo }}(X, Y) \hookrightarrow \mathcal{S}(X, Y)
$$

induces a bijective correspondence of the path-connected components of the two spaces.

Comparing with the Oka-Grauert principle [G3, Gr4, FP1] one might expect that the above inclusion is a weak homotopy equivalence; however, our proof does not give this much.

In the remainder of this section we discuss the question which manifolds satisfy Properties $S_{n}$ and $H S_{n}$. For Riemann surfaces both properties hold precisely on the non-hyperbolic ones (Corollary 2.8). For manifolds of dimension $>1$ a complete answer seems out of reach even in the class of projective algebraic surfaces. The following result gives some nontrivial examples. 
PROPOSITION 2.5. The following manifolds $Y^{q}$ satisfy Properties $S_{n}$ and $H S_{n}$ for any $n>q$, as well as for $n=q=1$ :

(a) $\mathbb{C}^{q}, \mathbb{C P}^{q}$, or a complex Grassmanian;

(b) a Zariski open subset in any of the manifolds from (a) whose complement contains no complex hypersurfaces.

For a proof see Sect. 5. The following simple observation will be useful.

PROPOSITION 2.6. Let $\pi: \widetilde{Y} \rightarrow Y$ be a holomorphic covering. If one of the manifolds $Y, \widetilde{Y}$ satisfies Property $S_{n}$ (resp. $H S_{n}$ ) then so does the other.

Proof. Assume first that $\widetilde{Y}$ satisfies $S_{n}$. Given a holomorphic submersion $f: O \rightarrow Y$ from a special compact convex set $O \subset \mathbb{C}^{n}$, there is a holomorphic lifting $g: O \rightarrow \widetilde{Y}$ (satisfying $\pi \circ g=f$ ) which is also a submersion since $\pi$ is locally biholomorphic. By the assumed Property $S_{n}$ of $\widetilde{Y}$ we can approximate $g$ by an entire submersion $\widetilde{g}: \mathbb{C}^{n} \rightarrow \widetilde{Y}$, and then $\widetilde{f}=\pi \circ \widetilde{g}: \mathbb{C}^{n} \rightarrow Y$ is a submersion such that $\left.\widetilde{f}\right|_{O}$ approximates $f$. This proves that $Y$ also satisfies $S_{n}$.

Conversely, assume that $Y$ satisfies $S_{n}$. Given a submersion $g: O \rightarrow \widetilde{Y}$, we approximate the submersion $f=\pi \circ g: O \rightarrow Y$ by a submersion $\tilde{f}: \mathbb{C}^{n} \rightarrow \underset{\widetilde{Y}}{Y}$ (using Property $S_{n}$ of $Y$ ) and then lift $\widetilde{f}$ to a (unique!) map $\widetilde{g}: \mathbb{C}^{n} \rightarrow \widetilde{Y}$ satisfying $\widetilde{g}\left(z_{0}\right)=g\left(z_{0}\right)$ for some point $z_{0} \in O$. Then $\widetilde{g}$ is a holomorphic submersion which approximates $g$ on $O$.

The same arguments hold for homotopies of submersions which gives the corresponding statement for Property $H S_{n}$. This proves Proposition 2.6.

Combining Propositions 2.5 and 2.6 we obtain

COROLLARY 2.7. A complex manifold $Y$ whose universal covering space is biholomorphic to $\mathbb{C}^{q}$, or to $\mathbb{C}^{q} \backslash A$ for an algebraic subvariety of codimension at least two, satisfies Properties $S_{n}$ and $H S_{n}$ when $n>q$ or $n \geq q=1$.

The manifolds covered by $\mathbb{C}^{q}$ are of the form $\mathbb{C}^{k} \times\left(\mathbb{C}^{*}\right)^{l} \times T^{s}(k+l+s=q)$, where $\mathbb{C}^{*}=\mathbb{C} \backslash\{0\}$ and $T^{s}$ is a complex torus, i.e., $T^{s}=\mathbb{C}^{s} / \Gamma$ for a lattice $\Gamma \subset \mathbb{C}^{s}$ of rank $2 s$. Recall that every Hopf manifold is a holomorphic quotient of $\mathbb{C}^{q} \backslash\{0\}$ and hence Theorem 2.1 applies (see [B, p. 172]).

The following result, which follows from Proposition 2.5 and Corollary 2.7, is a complete solution to the submersion problem if $Y$ is a Riemann surface.

COROLLARY 2.8. If $Y$ is any of the Riemann surfaces $\mathbb{C P}^{1}, \mathbb{C}, \mathbb{C}^{*}$, or a complex torus $\mathbb{C} / \Gamma$ then any continuous map $f_{0}: X \rightarrow Y$ from a Stein manifold $X$ is homotopic to a holomorphic submersion $f_{1}: X \rightarrow Y$. Furthermore, if $\left.f_{0}\right|_{K}: K \rightarrow Y$ is a holomorphic submersion for a compact $\mathcal{O}(X)$-convex subset $K \subset X$ then the homotopy can be chosen to appoximate $f_{0}$ uniformly on $K$. Conversely, if a Riemann surface $Y$ admits a nonconstant holomorphic map $\mathbb{C} \rightarrow Y$ then $Y$ belongs to the above list. 
Indeed, the universal covering of any Riemann surface is either $\mathbb{C P}^{1}, \mathbb{C}$ or the disc, and only the first two admit a nonconstant holomorphic image of $\mathbb{C}$; their holomorphic quotients are listed in Corollary 2.8.

The manifolds for which we prove Property $S_{n}$ in this paper are all subelliptic in the sense of [F3], i.e., they admit a finite dominating family of holomorphic sprays; hence by the main result in [F3] any continuous map $X \rightarrow Y$ is homotopic to a holomorphic map (the Oka-Grauert principle).

Problem 1. Does every subelliptic manifold $Y$ satisfy Property $S_{n}$ for all $n>\operatorname{dim} Y ?$

A good test case may be complex Lie groups $G$; a dominating holomorphic spray on $G$ is given by $s(g, v)=\exp (v) g$ where $g \in G$ and $v$ is a vector in the Lie algebra of $G$.

Problem 2. Does a manifold $Y$ which satisfies $S_{n}$ for some $n \geq \operatorname{dim} Y$ also satisfy $S_{k}$ for $k>n$ ?

Problem 3. Is the Property $S_{n}$ invariant with respect to proper modifications such as blow-ups and blow-downs?

\section{Holomorphic approximation on handlebodies.}

The main result of this section, Theorem 3.2, concerns holomorphic approximation of mappings between complex manifolds or, more generally, of sections of holomorphic submersions, on certain compact sets obtained by attaching a totally real submanifold to a compact holomorphically convex subset. Theorem 3.2, which generalizes a result of of Hörmander and Wermer [HW, Theorem 4.1], will be used in the proof of Theorem 2.1 in Sect. 4. The proof uses Hörmander's $L^{2}$-solution to the $\bar{\partial}$-equation [Hö1, Hö2] on special Stein neighborhoods of $S$ furnished by Theorem 3.1 below. For $X=\mathbb{C}^{n}$ Theorem 3.1 is due to Hörmander and Wermer [HW, Theorem 3.1]; here we extend it to arbitrary complex manifolds.

A compact set $K$ in a complex manifold $X$ is said to be holomorphically convex if $K$ has an open Stein neighborhood $\Omega$ in $X$ such that $K$ is $\mathcal{O}(\Omega)$ convex. By the classical theory (see e.g. Chapter 2 in [Hö2]) holomorphic convexity of $K$ is equivalent to the existence of a Stein neighborhood $\Omega$ of $K$ and a continuous (or smooth) plurisubharmonic function $\rho \geq 0$ on $\Omega$ such that $\rho^{-1}(0)=K$ and $\rho$ is strongly plurisubharmonic on $\Omega \backslash K$. We may take $\Omega=\left\{\rho<c_{1}\right\}$ for some $c_{1}>0$; for any $c \in\left(0, c_{1}\right)$ the sublevel set $\{\rho<c\} \subset \subset \Omega$ is then Stein and Runge in $\Omega$ (Sect. 4.3 in [Hö2]).

A $\mathcal{C}^{1}$ submanifold $M$ of a complex manifold $X$ is totally real in $X$ if for each $p \in M$ the tangent space $T_{p} M \subset T_{p} X$ contains no complex line of $T_{p} X$.

THEOREM 3.1. Let $X$ be a complex manifold. Assume that $S=K_{0} \cup M$ is a closed subset of an open set in $X$ such that $K_{0}$ is compact holomorphically convex and $M=S \backslash K_{0}$ is a $\mathcal{C}^{1}$ totally real submanifold. If there exists a 
compact holomorphically convex set $K_{1} \subset S$ which is a relative neighborhood of $K_{0}$ in $S$ then every compact set $K$ with $K_{0} \subset K \subset S$ is holomorphically convex. Fix any such $K$. Let $d$ be a distance function on $X$ induced by a smooth Riemannian metric. Fix a neighborhood $N$ of $K_{0}$ in $X$. There are a constant $C>1$ and for any sufficiently small $\epsilon>0$ a Stein domain $\omega_{\epsilon} \subset X$ such that

(i) $\omega_{\epsilon}$ contains all points of distance $<\epsilon$ from $K$,

(ii) all points $x \in \omega_{\epsilon} \backslash N$ have distance $<C \epsilon$ from $M$.

For $X=\mathbb{C}^{n}$ this is Theorem 3.1 in [HW] where the result is proved with $C=2$. In our application (Sect. 4) $K_{0}$ is the closure of a strongly pseudoconvex domain and $M$ is attached to $K_{0}$ along a submanifold $b M \subset b K_{0}=: \Sigma$ which

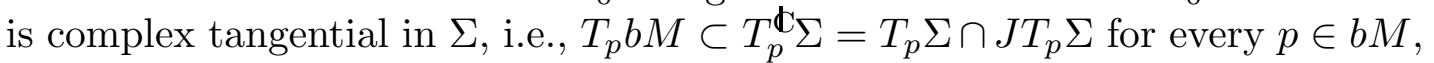
where $J$ is the almost complex structure operator on $T X$. For such $K_{0}$ and $M$, and with $X=\mathbb{C}^{n}$, results on holomorphic convexity of $K_{0} \cup M$ can be found in [E] (Lemmas 3.3.1. and 3.4.3.), [Ro, Lemma 2], [FK], and other papers.

Proof of Theorem 3.1. By the assumption on $K_{0}$ there is a smooth plurisubharmonic function $\rho_{0} \geq 0$ in an open Stein neighborhood $U_{0} \subset X$ of $K_{0}$ such that $\rho_{0}^{-1}(0)=K_{0}$. We may assume that the neighborhood $N$ of $K_{0}$ in Theorem 3.1 is chosen such that $N \subset U_{0}$ and $N \cap S \subset K_{1}$. Choose a sufficiently small $c_{2}>0$ such that $\left\{\rho_{0}<c_{2}\right\} \subset \subset N$. Also choose constants $0<c_{0}<c_{0}^{\prime}<c_{1}<c_{1}^{\prime}<c_{2}$.

Since Theorem 3.1 only concerns compact subsets of $S$, we may assume that $S$ is compact. Since $M$ is totally real, there is a $\mathcal{C}^{2}$ strongly plurisubharmonic function $\tau \geq 0$ in an open set $V_{0} \supset M$ which vanishes quadratically on $M$ [HW]. Replacing $\tau$ by $c \tau$ for a suitable $c>0$ and shrinking $V_{0}$ around $M$ if necessary we may assume that

$$
\tau(x)<d(x, M)^{2}, \quad x \in V_{0} .
$$

Since $\tau$ vanishes quadratically on $M$, there is a $C>0$ such that

$$
\tau(x)>d(x, M)^{2} / C^{2}, \quad x \in V_{0} \cap\left\{\rho_{0} \geq c_{0}\right\} .
$$

Recall that $K_{1}$ is assumed to be holomorphically convex. Choose a smooth plurisubharmonic function $\rho_{1} \geq 0$ in an open neighborhood $U_{1}$ of $K_{1}$ such that $\rho_{1}^{-1}(0)=K_{1}$ and $\rho_{1}$ is strongly plurisubharmonic off $K_{1}$. Since $\rho_{1}$ vanishes at least to second order at each point $x \in K_{1}$, we may assume (after rescaling $\rho_{1}$ if necessary) that

$$
\rho_{1}(x)<d(x, S)^{2} / C^{2}, \quad x \in U_{1} \cap\left\{\rho \leq c_{2}\right\}
$$

where $C$ is the constant from (3.2). Choose a smooth cut-off function $\chi \geq 0$ on $X$ which equals one on $\left\{\rho_{0} \leq c_{0}\right\}$ and satisfies supp $\chi \subset \subset\left\{\rho_{0}<c_{0}^{\prime}\right\}$. For a 
sufficiently small $\delta>0$ the function $\tau_{\delta}=\tau-\delta \chi$ is strongly plurisubharmonic on $V_{0} \cap\left\{\rho_{0} \geq c_{0}\right\}$ (we may need to shrink $V_{0}$ around $M$ ). Clearly $\tau_{\delta}=\tau$ on $V_{0} \cap\left\{\rho_{0} \geq c_{0}^{\prime}\right\}$. Fix such a $\delta$. Choose an open neighborhood $V \subset U_{1} \cup V_{0}$ of $S$ and let $\rho: V \rightarrow \mathbb{R}_{+}$be defined by

$$
\rho= \begin{cases}\rho_{1} & \text { on } V \cap\left\{\rho_{0}<c_{0}\right\}, \\ \max \left\{\rho_{1}, \tau_{\delta}\right\} & \text { on } V \cap\left\{c_{0} \leq \rho_{0} \leq c_{0}^{\prime}\right\}, \\ \tau & \text { on } V \cap\left\{\rho_{0}>c_{0}^{\prime}\right\} .\end{cases}
$$

It is easily verified that these choices are compatible provided that the neighborhood $V$ of $S$ is chosen sufficiently small. (When checking the compatibility near $M \cap\left\{\rho_{0}=c_{0}^{\prime}\right\}$ the reader should observe that, by (3.2) and (3.3), we have $\rho_{1}<\tau$ and hence $\rho=\tau$ there. Near $M \cap\left\{\rho_{0}=c_{0}\right\}$ we have $\rho_{1} \geq 0$ while $\tau_{\delta}<0$, hence $\rho=\rho_{1}$.) The function $\rho \geq 0$ is plurisubharmonic, strongly plurisubharmonic on $V \cap\left\{\rho_{0} \geq c_{0}^{\prime}\right\}$ (where it equals $\tau$ ), and $\rho^{-1}(0)=S$. For every sufficiently small $\epsilon>0$ the set

$$
\omega_{\epsilon}=\left\{x \in V: \rho(x)<\epsilon^{2}\right\} \subset \subset V
$$

is a pseudoconvex open neighborhood of $S$ which satisfies

$$
\{x \in X: d(x, S)<\epsilon\} \subset \omega_{\epsilon}, \quad \omega_{\epsilon} \backslash N \subset\{x \in X: d(x, M)<C \epsilon\} .
$$

The first inclusion follows from $\rho(x)<d(x, S)^{2}$ which is a consequence of $(3.1)$ and (3.3). The second inclusion is a consequence of (3.2) and the fact that $\rho=\tau$ on $V \backslash N$.

It remains to show that the sets $\omega_{\epsilon}$ are Stein. Fix $\epsilon$ and choose an increasing convex function $h_{\epsilon}:\left(-\infty, \epsilon^{2}\right) \rightarrow \mathbb{R}$ with $\lim _{t \rightarrow \epsilon^{2}} h(t)=+\infty$. Then $h_{\epsilon} \circ \rho$ is a plurisubharmonic exhaustion function on $\omega_{\epsilon}$. In order to obtain a strongly plurisubharmonic exhaustion function on $\omega_{\epsilon}$ we proceed as follows. Choose a smooth strongly plurisubharmonic function $\xi: U_{0} \rightarrow \mathbb{R}$ (such $\xi$ exists since $U_{0}$ is Stein). Also choose a smooth cut-off function $\chi \geq 0$ on $X$ such that $\chi=1$ on $\left\{\rho_{0} \leq c_{1}\right\}$ and $\operatorname{supp} \chi \subset \subset\left\{\rho_{0}<c_{1}^{\prime}\right\}$. If $\delta>0$ is chosen sufficiently small then $\widetilde{\rho}=\rho+\delta \chi \xi$ is strongly plurisubharmonic on $V$. Indeed, on $V \cap\left\{\rho_{0} \leq c_{1}\right\}$ we have $\widetilde{\rho}=\rho+\delta \xi$ which is strongly plurisubharmonic for every $\delta>0$; on $V \cap\left\{\rho_{0}>c_{1}\right\}$ the function $\rho$ is strongly plurisubharmonic and hence so is $\widetilde{\rho}$ provided that $\delta$ is chosen sufficiently small. For such $\delta$ the function $h_{\epsilon} \circ \rho+\widetilde{\rho}$ is a strongly plurisubharmonic exhaustion on $\omega_{\epsilon}$ and hence $\omega_{\epsilon}$ is Stein.

This gives a desired Stein neighborhood basis $\omega_{\epsilon}$ of $S$ satisfying Theorem 3.1. The same proof applies to any compact subset $K \subset S$ containing $K_{0}$. Alternatively one can apply the above proof with $\rho$ replaced by $\rho+\tau_{K}$ where $\tau_{K} \geq 0$ is a smooth function which vanishes to order $>2$ on $K$. This completes the proof of Theorem 3.1.

Remark. Since the function $\rho$ constructed above is plurisubharmonic on the Stein manifold $\omega_{\epsilon_{0}}=\left\{\rho<\epsilon_{0}\right\}$ for some small $\epsilon_{0}>0$, its sublevel sets $\omega_{\epsilon}=\{\rho<\epsilon\}$ for $\epsilon \in\left(0, \epsilon_{0}\right)$ are Runge in $\omega_{\epsilon_{0}}$. 
THEOREM 3.2. Let $K_{0}$ and $S=K_{0} \cup M$ be compact holomorphically convex subsets in a complex manifold $X$ such that $M=S \backslash K_{0}$ is a totally real $m$-dimensional submanifold of class $\mathcal{C}^{r}$. Assume that $r \geq m / 2+1$ and let $k$ be an integer satisfying $0 \leq k \leq r-m / 2-1$. Given an open set $U \subset X$ containing $K_{0}$ and a map $f: U \cup M \rightarrow Y$ to a complex manifold $Y$ such that $\left.f\right|_{U}$ is holomorphic and $\left.f\right|_{M} \in \mathcal{C}^{r}(M)$, there exist open sets $V_{j} \subset X$ containing $S$ and holomorphic maps $f_{j}: V_{j} \rightarrow Y(j=1,2,3, \ldots)$ such that, as $j \rightarrow \infty$, the sequence $f_{j}$ converges to $f$ uniformly on $K_{0}$ and in the $\mathcal{C}^{k}$-sense on $M$. If in addition $X_{0}$ is a closed complex subvariety of $X$ which does not intersect $M$ and $s \in \mathbb{N}$ then we can choose the approximating sequence such that $f_{j}$ agrees to order $s$ with $f$ along $X_{0} \cap V$ for all $j=1,2,3, \ldots$. The analogous result holds for sections $f: X \rightarrow Z$ of any holomorphic submersion $h: Z \rightarrow X$.

The domains $V_{j}$ of $f_{j}$ in Theorem 3.2 may shrink to $S$ as $j \rightarrow \infty$. Although none of the manifolds $X, Y, Z$ in Theorem 3.2 is assumed to be Stein, we shall reduce the proof to that case. In our applications $K_{0}$ will be a sublevel set $\{\rho \leq c\}$ of a smooth strongly plurisubharmonic function on $X$ and $M$ will be a smooth totally real handle attached to the hypersurface $b K_{0}=\{\rho=c\}$ along a legendrian (complex tangential) sphere.

Proof. When $X=\mathbb{C}^{n}, Y=\mathbb{C}$ (i.e., $f$ is a function) and $k=0$, Theorem 3.2 coincides with Theorem 4.1 in [HW, p. 11]. For later purposes we recall the sketch of proof which uses the Stein neighborhood basis $\omega_{\epsilon} \subset X$ of $S$ furnished by Theorem $3.1 \mathrm{in}[\mathrm{HW}]$ (compare with Theorem 3.1 above). One first obtains a $\mathcal{C}^{r}$ extension $u$ of $f$ in a neighborhood of $M$ such that $\left|D^{s}(\bar{\partial} u)\right|=o\left(d_{M}^{r-1-s}\right)$, where $d_{M}$ denotes the distance to $M$ and $D^{s}$ is the total derivative of order $s \leq r-1$ (Lemma 4.3 in [HW]). Then one solves $\bar{\partial} w_{\epsilon}=u$ with $L^{2}$ estimate on $\omega_{\epsilon}[$ Hö1, Hö2]. By the interior elliptic regularity of $\bar{\partial}$ this implies the uniform estimate $\left|w_{\epsilon}\right|=o\left(\epsilon^{r-m / 2-1}\right)=o\left(\epsilon^{k}\right)$ on $\omega_{c \epsilon}$ for any fixed $c \in(0,1)$ (the second display on p. 16 in [HW]). If $0<s \leq k$ we also have $\left|D^{s} w_{\epsilon}\right|=o\left(\epsilon^{k-s}\right)$ on $\omega_{c^{\prime} \epsilon}$ for a fixed $c^{\prime} \in(0, c)$ (see e.g. Lemma 3.2 and the proof of Proposition 2.3 in [FL]). The function $f_{\epsilon}=u-w_{\epsilon}$ is holomorphic on $\omega_{\epsilon}$; as $\epsilon \rightarrow 0, f_{\epsilon}$ converges to $f$ uniformly on $K_{0}$ and in the $\mathcal{C}^{r}$-sense on $M$. Since $\omega_{\epsilon}$ is Runge in $V:=\omega_{\epsilon_{0}}$ (see the Remark preceding Theorem 3.2), we can approximate $f_{\epsilon}$ by $F_{\epsilon}: V \rightarrow \mathbb{C}$ and obtain the desired approximating sequence on a fixed open set $V \subset \mathbb{C}^{n}$. This proves the special case of Theorem 3.2.

For $Y=\mathbb{C}^{N}$ the result follows immediately by applying it componentwise. The case when $X$ is a Stein manifold (and $Y=\mathbb{C}^{N}$ ) reduces to the special case by embedding $X$ as a closed complex submanifold in some $\mathbb{C}^{n}$, or by applying the proof in $[\mathrm{HW}]$ with the Stein neighborhood basis furnished by Theorem 3.1 above. To prove the general case of Theorem 3.2 we need the following.

LEMMA 3.3. Let $h: Z \rightarrow X$ be a holomorphic submersion of a complex manifold $Z$ to a complex manifold $X$. Assume that $S=K_{0} \cup M \subset X$ satisfies the hypotheses of Theorem 3.2. Let $U$ be an open set in $X$ containing $K_{0}$ and let $f: U \cup M \rightarrow Z$ be a section which is holomorphic on $U$ and smooth of class 
$\mathcal{C}^{1}$ on $M$. Then $f(S)$ has a Stein neighborhood basis in $Z$.

Proof. We may assume that $U$ is Stein and $K_{0}$ is $\mathcal{O}(U)$-convex. The submanifold $f(M) \subset Z$ is projected by $h$ bijectively onto the totally real submanifold $M \subset X$ and hence $f(M)$ is totally real in $Z$. Since $\left.f\right|_{U}$ is holomorphic, $f(U)$ is a closed complex submanifold of $\left.Z\right|_{U}=h^{-1}(U)$ and hence by [De, S] it has an open Stein neighborhood $\left.\widetilde{U} \subset Z\right|_{U}$. For any compact $\mathcal{O}(U)$-convex subset $K \subset \subset U$ the set $f(K)$ is holomorphically convex in $f(U)$ and hence (since $f(U)$ is a closed complex submanifold of $\widetilde{U}$ ) also in $\widetilde{U}$. Applying this with $K=K_{0}$, and also with $K=\left(K_{0} \cup S\right) \cap N$ for some compact neighborhood $N \subset U$ of $K_{0}$, we see that $f(S) \subset Z$ satisfies the hypothesis of Theorem 3.1 and hence it has a basis of Stein neighborhoods.

We continue with the proof of Theorem 3.2. Assume that $f: U \cup M \rightarrow Z$ is a section of $h: Z \rightarrow X$ which is holomorphic in $U$ and of class $\mathcal{C}^{r}$ on $M$ for some $r \geq 1$. Fix a Stein neighborhood $\Omega \subset Z$ of $f(S)$ furnished by Lemma 3.3 and embed $\Omega$ as a closed complex submanifold of a Euclidean space $\mathbb{C}^{N}$. There is an open neighborhood $\widetilde{\Omega} \subset \mathbb{C}^{N}$ of $\Omega$ and a holomorphic retraction $\phi: \widetilde{\Omega} \rightarrow \Omega$ [DG]. We consider $f$ as a map into $\mathbb{C}^{N}$ via the embedding $\Omega \hookrightarrow \mathbb{C}^{N}$. The special case of Theorem 3.2 gives a sequence of holomorphic maps $g_{j}: V \rightarrow \mathbb{C}^{N}(j \in \mathbb{N})$ in an open neighborhood $V \subset X$ of $S$ such that $\left.\lim _{j \rightarrow \infty} g_{j}\right|_{S}=\left.f\right|_{S}$ (the convergence is uniform on $K_{0}$ and in the $\mathcal{C}^{k}$-sense on $\left.M\right)$. Let $V_{j}=\left\{x \in V: g_{j}(x) \in \widetilde{\Omega}\right\}$; this is a neighborhood of $S$ for sufficiently large $j \in \mathbb{N}$. The sequence of maps $\widetilde{f}_{j}=\phi \circ g_{j}: V_{j} \rightarrow \Omega$ then satisfies the conclusion of Theorem 3.2 except that $\widetilde{f}_{j}$ need not be a section of $h$. This is corrected by projecting the point $\tilde{f}_{j}(x)$ to the fiber $Z_{x}=h^{-1}(x)$ by the holomorphic retraction provided by the following

LEMMA 3.4. Let $\Omega$ be a Stein manifold and $h: \Omega \rightarrow V$ a holomorphic submersion onto a complex manifold $V$. There are an open Stein set $W \subset V \times \Omega$ containing $\Gamma:=\{(x, z) \in V \times \Omega: h(z)=x\}$ and a holomorphic retraction $\pi: W \rightarrow \Gamma$ such that $\pi(x, z)=\left(x, \pi_{2}(x, z)\right)$ for every $(x, z) \in W$.

It follows that $h\left(\pi_{2}(x, z)\right)=x$, i.e., $\pi_{2}(x, \cdot)$ is a holomorphic retraction of an open neighborhood of the fiber $h^{-1}(x)$ in $\Omega$ onto $h^{-1}(x)$ for every fixed $x \in V$. Assuming Lemma 3.4 for a moment we set $f_{j}(x)=\pi_{2}\left(x, \tilde{f}_{j}(x)\right)$ for $j=1,2, \ldots$; these are holomorphic sections of $h$ in small open neighborhoods $V_{j} \subset X$ of $S$ satisfying Theorem 3.2 .

The version of Theorem 3.2 with interpolation along a subvariety $X_{0}$ not intersecting $M=S \backslash K_{0}$ can be satisfied by using an embedding $\Omega \hookrightarrow \mathbb{C}^{N}$ and writing $f=f_{0}+\sum_{l=1}^{l_{0}} h_{l} g_{l}$ in a neighborhood $V \subset X$ of $S$, where $f_{0}$ and $g_{l}$ are maps $V \rightarrow \mathbb{C}^{N}$ such that $f_{0}$ is holomorphic in $V, g_{l} \in \mathcal{C}^{r}(V)$ is holomorphic over a neighborhood of $K_{0}$ (where $f$ is holomorphic), and the functions $h_{1}, \ldots, h_{l_{0}} \in \mathcal{O}(V)$ vanish to order $s$ on $X_{0}$ and satisfy $X_{0} \cap V=$ $\left\{x \in V: h_{l}(x)=0,1 \leq l \leq l_{0}\right\}$. (See Lemma 8.1 in [FP2, p. 660] for the details.) Applying Theorem 3.2 with $Y=\mathbb{C}^{N}$ we approximate each $g_{l}$ on $S$ by a sequence of sections $g_{l, j}(j=1,2,3, \ldots)$ holomorphic in a neighborhood 
of $S$ in $X$. This gives a holomorphic sequence $\tilde{f}_{j}=f_{0}+\sum_{l=1}^{l_{0}} h_{l} g_{l, j}$ whose restriction to $S$ converges to $f$ as $j \rightarrow \infty$ (in the sense of Theorem 3.2). It remains to compose $\tilde{f}_{j}$ with the two retractions as above (first by the retraction $\phi$ onto $\Omega$ and then by the retraction furnished by Lemma 3.4) to get a sequence of holomorphic sections $f_{j}: V_{j} \rightarrow Z(j=1,2, \ldots)$ which agree with $f$ to order $s$ on $X_{0}$ and satisfy $\left.\left.f_{j}\right|_{S} \rightarrow f\right|_{S}$ as $j \rightarrow \infty$. This completes the proof of Theorem 3.2 granted that Lemma 3.4 holds.

Proof of Lemma 3.4. Clearly $\Gamma$ is a closed complex submanifold of $V \times \Omega$. Let $p_{1}: V \times \Omega \rightarrow V, p_{2}: V \times \Omega \rightarrow \Omega$ denote the respective projections. Observe that $\left.p_{2}\right|_{\Gamma}: \Gamma \rightarrow \Omega$ is bijective with the inverse $z \rightarrow(h(z), z)$; hence $\Gamma$ is Stein and consequently it has an open Stein neighborhood in $V \times \Omega$.

Consider the holomorphic vector subbundle $E \subset T(V \times \Omega)$ whose fiber over $(x, z)$ consists of all vectors $(0, \xi), \xi \in T_{z} \Omega$. The restricted bundle $\left.E\right|_{\Gamma}$ can be decomposed as $\left.E\right|_{\Gamma}=E_{0} \oplus N$ where $E_{0}=\left.E\right|_{\Gamma} \cap T \Gamma$ and $N$ is some complementary subbundle. Observe that $N$ is the normal bundle of $\Gamma$ in $V \times \Omega$ (here we use the hypothesis that $h: \Omega \rightarrow V$ is a submersion). Let $N_{0}$ denote the zero section of $N$. By the Docquier-Grauert theorem [DG] there is a biholomorphic map $\Phi$ from an open neighborhood $U \subset N$ of $N_{0}$ onto an open neighborhood $W \subset V \times \Omega$ of $\Gamma$ which maps $N_{0}$ onto $\Gamma$ and maps the fiber $N_{(x, z)} \cap U$ to $\{x\} \times \Omega=p_{1}^{-1}(x)$ for any $(x, z) \in \Gamma$. (We can obtain such $\Phi$ by composing local flows of holomorphic vector fields which are tangent to $N$.) Choosing $U$ to have convex fibers it follows that $\Phi$ conjugates the base projection $U \rightarrow N_{0}$ to a holomorphic retraction $\pi: W \rightarrow \Gamma$ satisfying the conclusion of Lemma 3.4.

Remark. The loss of smoothness in Theorem 3.2 is due to the method of proof. When $K_{0}=\emptyset$ and $S$ is a totally real submanifold of $X$ of class $\mathcal{C}^{r}$, the optimal $\mathcal{C}^{r}$-to- $\mathcal{C}^{r}$ approximation was proved by Range and Siu [RS] following the work of many authors. The kernel approach developed in [FL $\varnothing]$ gives precise approximation of smooth diffeomorphisms by biholomorphisms in tubular neighborhoods. Both approaches can likely be adapted to the situation considered here, but we do not need such sharp approximation.

\section{Proof of Theorem 2.1.}

We first state parts (a) and (b) of Theorem 2.1 in a precise quantitative form; part (c) will be considered later.

THEOREM 4.1. Let $X$ be a Stein manifold, $K \subset X$ a compact $\mathcal{O}(X)$ convex subset and $Y$ a complex manifold with $\operatorname{dim} Y \leq \operatorname{dim} X$. Choose a distance function $d$ on $Y$ induced by a complete Riemannian metric. Assume that $\left(f_{0}, \iota_{0}\right) \in \mathcal{S}(X, Y)$ is such that $\left.f_{0}\right|_{K}: K \rightarrow Y$ is a holomorphic submersion and $\left.\iota_{0}\right|_{K}=\left.T f_{0}\right|_{K}$. If $Y$ satisfies Property $S_{n}$ with $n=\operatorname{dim} X$ then for every $\epsilon>0$ there is a homotopy $\left(f_{t}, \iota_{t}\right) \in \mathcal{S}(X, Y)(t \in[0,1])$ from $\left(f_{0}, \iota_{0}\right)$ to some $\left(f_{1}, T f_{1}\right) \in \mathcal{S}_{\text {holo }}(X, Y)$ such that for every $t \in[0,1],\left.f_{t}\right|_{K}: K \rightarrow Y$ is a holomorphic submersion, $\left.T f_{t}\right|_{K}=\left.\iota_{t}\right|_{K}$, and $\sup _{x \in K} d\left(f_{t}(x), f_{0}(x)\right)<\epsilon$. 
Proof. We shall follow the construction of holomorphic submersions $X \rightarrow \mathbb{C}^{q}$ in [F1], indicating the necessary changes and additional arguments.

Assume that $U \subset X$ is an open set containing $K$ such that $\left.f_{0}\right|_{U}: U \rightarrow Y$ is a holomorphic submersion and $\left.T f_{0}\right|_{U}=\left.\iota_{0}\right|_{U}$. Choose a smooth strongly plurisubharmonic Morse exhaustion function $\rho$ on $X$ such that $K \subset\{\rho<$ $0\} \subset \subset U$ and 0 is a regular value of $\rho$. We may assume furthermore that in some local holomorphic coordinates near each critical point the function $\rho$ is a quadratic normal form given by (6.1) in [F1].

The construction of the homotopy $\left(f_{t}, \iota_{t}\right) \in \mathcal{S}(X, Y)$ is done in a countable sequence of stages, and each stage consists of finitely many steps. We use two different types of steps, one for crossing the noncritical levels of $\rho$ and the other one for crossing a critical level. The selection of sets involved in stages and steps is done in advance and depends only on the exhaustion function $\rho$. On the other hand, some of the constants in the approximation at each stage (or step) are chosen inductively and depend on the partial solution obtained in the previous steps. The entire construction is quite similar to the proof of the Oka-Grauert principle in [HL2], and especially in [FP1]. We first explain the global scheme; compare with Sect. 6 in [F1].

Let $p_{1}, p_{2}, p_{3}, \ldots$ be the critical points of $\rho$ in $\{\rho>0\} \subset X$, ordered so that $0<\rho\left(p_{1}\right)<\rho\left(p_{2}\right)<\rho\left(p_{3}\right)<\ldots$. Choose a sequence $0=c_{0}<c_{1}<c_{2}<\ldots$ with $\lim _{j \rightarrow \infty} c_{j}=+\infty$ such that $c_{2 j-1}<\rho\left(p_{j}\right)<c_{2 j}$ for every $j=1,2, \ldots$, and the numbers $c_{2 j-1}, c_{2 j}$ are close to $\rho\left(p_{j}\right)$; the desired closeness will be specified below when crossing the critical level $\left\{\rho=\rho\left(p_{j}\right)\right\}$. If there are only finitely many $p_{j}$ 's, we choose the remainder of the sequence $c_{j}$ arbitrarily with $\lim _{j \rightarrow \infty} c_{j}=+\infty$. We subdivide the parameter interval of the homotopy into subintervals $I_{j}=\left[t_{j}, t_{j+1}\right]$ with $t_{j}=1-2^{-j}(j=0,1,2, \ldots)$.

In the $j$-th stage of the construction we assume inductively that we have a partial solution $\left(f_{t}, \iota_{t}\right) \in \mathcal{S}(X, Y)$ for $t \in\left[0, t_{j}\right]$ satisfying

$$
\sup \left\{d\left(f_{t}(x), f_{0}(x)\right): x \in K\right\}<\epsilon_{j}, \quad t \in\left[0, t_{j}\right]
$$

for some $\epsilon_{j} \leq \epsilon\left(1-2^{-j-1}\right)$, such that $f_{t_{j}}:\left\{\rho \leq c_{j}\right\} \rightarrow Y$ is a holomorphic submersion and $\iota_{t_{j}}=T f_{t_{j}}$ on $\left\{\rho \leq c_{j}\right\}$. For $j=0$ these conditions are satisfied with $\epsilon_{0}=\epsilon / 2$.

Choose a number $\delta_{j} \in\left(0, \epsilon_{j} 2^{-j-1}\right)$ such that any holomorphic map from $\left\{\rho \leq c_{j}\right\}$ to $Y$ which is uniformly $\delta_{j}$-close to $f_{t_{j}}$ (measured by the metric $d$ on $Y$ ) is a submersion on $\left\{\rho \leq c_{j-1}\right\}$. (For $j=0$ we choose $c_{-1}<0$ sufficiently close to 0 such that $K \subset\left\{\rho<c_{-1}\right\}$.) We also insure that $\delta_{j}<\delta_{j-1} / 2$ where $\delta_{j-1}$ is the analogous number from the previous stage; this condition is vacuous for $j=0$. The goal of the $j$-th stage is to extend the solution $\left(f_{t}, \iota_{t}\right) \in \mathcal{S}(X, Y)$ to the interval $t \in I_{j}=\left[t_{j}, t_{j+1}\right]$ (keeping it unchanged for $t \in\left[0, t_{j}\right]$ ) such that

$$
\sup \left\{d\left(f_{t}(x), f_{t_{j}}(x)\right): x \in X, \rho(x) \leq c_{j}\right\}<\delta_{j} / 2, \quad t \in I_{j},
$$


$T f_{t}=\iota_{t}$ on $\left\{\rho \leq c_{j}\right\}$ for every $t \in I_{j}$, and $T f_{t_{j+1}}=\iota_{t_{j+1}}$ on $\left\{\rho \leq c_{j+1}\right\}$. Then $f_{t_{j+1}}$ satisfies the inductive hypothesis on the set $\left\{\rho \leq c_{j+1}\right\}$ with $\epsilon_{j+1}=$ $\epsilon_{j}+\delta_{j} / 2<\epsilon\left(1-2^{-j-2}\right)$, thus completing the $j$-th stage.

Assume for a moment that this process can be worked out. For every $t \in\left[t_{j}, 1\right)$ the map $f_{t}$ is holomorphic on $\left\{\rho \leq c_{j}\right\}$ and $\iota_{t}=T f_{t}$ there. By (4.2) the limits $f_{1}=\lim _{t \rightarrow 1} f_{t}: X \rightarrow Y$ and $\iota_{1}=\lim _{t \rightarrow 1} \iota_{t}=\lim _{t \rightarrow 1} T f_{t}: T X \rightarrow T Y$ exist uniformly on compacts in $X$. It follows that $f_{1}: X \rightarrow Y$ is holomorphic and $\iota_{1}=T f_{1}$ on $X$. By the construction we also have

$$
d\left(f_{1}(x), f_{t_{j}}(x)\right)<\delta_{j}, \quad x \in\left\{\rho \leq c_{j}\right\}, j=0,1,2, \ldots
$$

The choice of $\delta_{j}$ implies that $f_{1}$ is a holomorphic submersion on $\left\{\rho \leq c_{j-1}\right\}$. Since this holds for every $j \in \mathbb{N}, f_{1}$ is a holomorphic submersion of $X$ to $Y$ and hence $\left(f_{1}, T f_{1}\right) \in \mathcal{S}_{\text {holo }}(X, Y)$. From (4.1) we also get $d\left(f_{1}(x), f_{0}(x)\right)<\epsilon$ for $x \in K$. This will complete the proof of Theorem 4.1 provided that we prove the inductive stage.

We first consider the noncritical stages, i.e., those for which $\rho$ has no critical values on $\left[c_{j}, c_{j+1}\right]$. (In our notation this happens for even values of $j$.) We solve the problem in finitely many steps of the following kind. We have compact subsets $A, B$ of $X$ satisfying

(i) $A, B, C:=A \cap B$, and $\widetilde{A}:=A \cup B$ are (the closures of) strongly pseudoconvex domains in $X$,

(ii) $\overline{A \backslash B} \cap \overline{B \backslash A}=\emptyset$ (the separation property), and

(iii) there is an open set $U$ in $X$ containing $B$ and a biholomorphic map $\psi: U \rightarrow$ $U^{\prime}$ onto an open subset $U^{\prime} \subset \mathbb{C}^{n}$ containing the cube $Q$ (2.1) such that $\psi(B)$ is a convex subset of $Q$ and $O:=\psi(A \cap U) \cap Q$ is a special convex set of the form $(2.2)$.

PROPOSITION 4.2. Let $A, B \subset X$ satisfy the properties (i)-(iii) above. Assume that $f: A \rightarrow Y$ is a holomorphic submersion. If $Y$ satisfies Property $S_{n}$ with $n=\operatorname{dim} X$ then for any $\delta>0$ there is a homotopy of holomorphic submersions $f_{t}: A \rightarrow Y(t \in[0,1])$, with $f_{0}=f$, such that $\sup _{x \in A} d\left(f_{t}(x), f(x)\right)<\delta$ for every $t \in[0,1]$ and $f_{1}$ extends to a holomorphic submersion $A \cup B \rightarrow Y$.

For $Y=\mathbb{C}^{q}$ with $q<\operatorname{dim} X$ this is Lemma 6.3 in [F1]. Its proof also applies in our situation, except that we must replace the use of Proposition 3.3 in [F1] by the assumed Property $S_{n}$ of $Y$. We include a sketch of proof.

By hypothesis the map $f \circ \psi^{-1}: U^{\prime} \rightarrow Y$ is a holomorphic submersion on $O=\psi(A \cap U) \cap Q \subset \mathbb{C}^{n}$. By Property $S_{n}$ of $Y$ we can approximate it uniformly on some fixed neighborhood of $O$ as close as desired by a holomorphic submersion $\widetilde{g}: Q \rightarrow Y$. (We actually apply $S_{n}$ with the pair $(r O, r Q)$ of dilated sets for some $r>1$ close to 1 in order to get approximation on a neighborhood of $O$.) Since $\psi(B) \subset Q$, the map $g=\widetilde{g} \circ \psi: B \rightarrow Y$ is a holomorphic submersion which approximates $f$ uniformly in a neighborhood of $A \cap B$ as close as desired. 
By Lemma 5.1 in [F1] we have $f=g \circ \gamma$ for a biholomorphic map $\gamma$ defined in an open neighborhood of $A \cap B$ in $X$ and uniformly very close to identity map. (Its distance from the identity only depends on the distance of $f$ and $g$ on the given neighborhood of $A \cap B$. The proof of Lemma 5.1 in [F1] holds for arbitrary target manifold $Y$.) If the approximations are sufficiently close, Theorem 4.1 in [F1] provides a decomposition $\gamma=\beta \circ \alpha^{-1}$ in a neighborhood of $A \cap B$, where $\alpha$ is a biholomorphic map close to the identity in a neighborhood of $A$ in $X$ and $\beta$ is a biholomorhic map close to the identity in a neighborhood of $B$. Thus $f \circ \alpha=g \circ \beta$ in a neighborhood of $A \cap B$, and hence the two sides define a holomorphic submersion $\widetilde{f}: A \cup B \rightarrow \mathbb{C}^{q}$ which approximates $f$ on $A$. Furthermore, there is a homotopy $\alpha_{t}(t \in[0,1])$ of biholomorphic maps close to the identity in some fixed neighborhood of $A$ such that $\alpha_{0}$ is the identity and $\alpha_{1}=\alpha$. (It suffices to embed $X$ as a complex submanifold in some Euclidean space, take the convex linear combinations of $\alpha$ with the identity map, and project this homotopy back to the submanifold $X$ by a holomorphic retraction.) Then $f_{t}:=f \circ \alpha_{t}: A \rightarrow Y$ is a homotopy of holomorphic submersions from $f_{0}=f$ to $f_{1}=\widetilde{f}$ satisfying Proposition 4.2. This completes the proof.

It remains to explain how Proposition 4.2 is used in the $j$-th stage of the construction. Since $\rho$ is assumed to have no critical values in $\left[c_{j}, c_{j+1}\right]$, we can obtain the set $\left\{\rho \leq c_{j+1}\right\}$ from $\left\{\rho \leq c_{j}\right\}$ by finite number of attachings of 'convex bumps' of the type introduced just before Proposition 4.2. We begin with $A_{0}=\left\{\rho \leq c_{j}\right\}$ and attach a bump $B_{0}$ to get $A_{1}=A_{0} \cup B_{0}$; then we attach a new bump $B_{1}$ to $A_{1}$ to obtain $A_{2}=A_{1} \cup B_{1}$, etc., until reaching $A_{k_{j}}=\left\{\rho \leq c_{j+1}\right\}$ (see Lemma 12.3 in [HL1]). The required number of steps $k_{j}$ depends only on $\rho$. We also subdivide the parameter interval $I_{j}=\left[t_{j}, t_{j+1}\right]$ into adjacent subintervals $I_{j, k}=\left[t_{j, k-1}, t_{j, k}\right]\left(k=1,2, \ldots, k_{j}\right)$ of equal length, one for every step.

Assume inductively that for some $1 \leq k<k_{j}$ a solution $\left(f_{t}, \iota_{t}\right) \in \mathcal{S}(X, Y)$ has already been defined for $t \in\left[0, t_{j, k-1}\right]$ such that $f:=f_{t_{j, k-1}}$ is a holomorphic submersion from $A_{k-1}$ to $Y$ and $\iota:=\iota_{t_{j, k-1}}=T f$ on this set. Applying Proposition 4.2 on $A=A_{k-1}$ we extend the family of solutions to the next subinterval $t \in I_{j, k}$ such that

$$
\sup \left\{d\left(f_{t}(x), f_{t_{j, k-1}}(x): x \in A_{k-1}\right\}<\delta_{j} / 2 k_{j}\right.
$$

(compare with (4.2)). We can also define $\iota_{t}$ for $t \in I_{j, k}$ such that $\iota_{t}=T f_{t}$ on $A_{k-1}, \iota_{t_{j, k}}=T f_{j, k}$ on $A_{k}, \iota_{t}$ is homotopic to $\iota_{0}$ and agrees with $\iota_{0}$ outside of some small neighborhood of $A_{k}$. In $k_{j}$ steps we extend the family of solutions $\left(f_{t}, \iota_{t}\right)$ to $t \in I_{j}$ and thus complete the $j$-th stage.

It remains to consider the critical stages, i.e., those for which $\rho$ has a critical point $p$ with $c_{j}<\rho(p)<c_{j+1}$. For $Y=\mathbb{C}^{q}$ this is explained in sections $6.2-6.4$ in $[F 1]$. Since the proof needs a few modifications, we shall go through it step by step.

Write $c=c_{j}$. We may assume that $c$ has been chosen as close to $\rho(p)$ as will be needed in the sequel. Near $p$ we use local holomorphic coordinates 
on $X$ in which $p=0$ and $\rho$ is a quadratic normal form (see (6.1) in [F1]). In particular, the stable manifold of $p=0$ for the gradient flow of $\rho$ is $\mathbb{R}^{\nu} \subset \mathbb{C}^{n}$ (the subspace spanned by the real parts of the first $\nu$ variables), where $\nu$ is the Morse index of $\rho$ at $p$. To cross the critical level $\rho=\rho(p)$ we perform the following three steps.

Step 1: Extension to a handle. We attach to $\{\rho \leq c\}$ the disc $M \subset \mathbb{R}^{\nu} \subset$ $\mathbb{C}^{n}$ (in the local coordinates) such that the attaching sphere $b M \subset\{\rho=c\}$ is complex tangential in the latter hypersurface (Subsect. 6.2 in [F1]). Let $(f, \iota)$ be the partial solution obtained after the first $j-1$ stages, so $f$ is a holomorphic submersion from a neighborhood of $\{\rho \leq c\}$ to $Y$ and $\iota=T f$ there.

LEMMA 4.3. There is a neighborhood $U$ of $S:=\{\rho \leq c\} \cup M$ in $X$ and a smooth map $g: U \rightarrow Y$ which agrees with $f$ in a neighborhood of $K_{0}:=$ $\{\rho \leq c\}$ such that for every $x \in M$ the differential $d g_{x}: T_{x} X \rightarrow T_{g(x)} Y$ is a surjective $\mathbb{C}$-linear map. Furthermore, $(f, \iota)$ can be connected to $(g, T g)$ by a path (homotopy) in $\mathcal{S}(U, Y)$ which is fixed in a neighborhood of $K_{0}$.

Proof. The main point is the extension of $f$ and its 1-jet to the handle $M$ such that the above properties are satisfied. One uses Gromov's convex integration lemma ([Gr2]; Section 2.4 of [Gr3], especially (D) and (E) in [Gr3, 2.4.1.]; Sect. 18.2 in [EM], especially Corollary 18.2.2.). The details given in [F1] (Lemmas 6.4 and 6.5) for the case $Y=\mathbb{C}^{q}$ remain valid for arbitrary target manifold $Y$. The differential relation controlling the problem is ample in the coordinate directions and hence Gromov's lemma applies.

It is (only) at this point of the proof that we use the hypothesis on the existence of a fiberwise surjective map $\iota: T X \rightarrow T Y$ with base map $f$. When $q=\operatorname{dim} Y \leq\left[\frac{n+1}{2}\right]$ it suffices to apply Thom's jet transversality theorem as in [F1], and in this case $\iota$ automatically exists.

Step 2: Holomorphic approximation on a handlebody. We denote the result of Step 1 again by $(f, \iota)$; thus $f$ is a holomorphic submersion from a neighborhood of the strongly pseudoconvex domain $K_{0}=\{\rho \leq c\} \subset X$ to $Y$, it is smooth in a neighborhood of the handle $M, d f_{x}: T_{x} X \rightarrow T_{f(x)} Y$ is surjective and $\mathbb{C}$-linear at each point $x \in M$, and $\iota_{x}=d f_{x}$ for every $x \in S=K_{0} \cup M$.

By [F1, Subsect. 6.3] the pair $\left(K_{0}, M\right)$ satisfies the hypothesis of Theorem 3.2 above, and hence there is a holomorphic map $\widetilde{f}$ from a neighborhood of $S$ to $Y$ which approximates $f$ uniformly on $K_{0}$ and in the $\mathcal{C}^{1}$-sense on $M$ as close as desired. (In [F1] the corresponding approximation result was proved for $Y=\mathbb{C}^{q}$.) If the approximations are sufficiently close then $\widetilde{f}$ is a holomorphic submersion from some open neighborhood of $S$ to $Y$. We patch the new map with the old one outside of a neighborhood of $S$.

Step 3: Crossing the critical level of $\rho$. We denote the result of Step 2 again by $f$ and set $\iota=T f$ in a neighborhood of $S=\{\rho \leq c\} \cup M$. We patch 
$\iota$ with $\iota_{0}$ outside of a neighborhood of $S$ by using a cut-off function in the parameter of the homotopy connecting $\iota$ to $\iota_{0}$.

By the scheme explained in [F1, Subsect. 6.4] we can approximately extend $f$ across the critical level of $\rho$ at $p$ by performing a noncritical stage with another strongly plurisubharmonic function $\tau$ furnished by Lemma 6.7 in [F1]. Afterwards we revert back to the original exhaustion function $\rho$ and proceed to the next noncritical stage associated with $\rho$. When changing the domain of the solution (first from a neighborhood of the handlebody $S$ to a suitable sublevel set of $\tau$, and later from a higher sublevel set of $\tau$ to a supercritical sublevel set $\rho$ ) we sacrifice a part of the domain, but the loss is compensated in the next stage. The approximation conditions for the given stage can be satisfied with the appropriate choices of constants at every step. No further changes from [F1] are needed apart from those already explained for a noncritical stage. This completes the proof of Theorem 4.1 and hence of (a), (b) in Theorem 2.1.

To prove part (c) of Theorem 2.1 we perform the same construction for homotopies of submersions connecting a given pair $f_{0}, f_{1}: X \rightarrow Y$. Property $H S_{n}$ is needed to obtain the analogue of Proposition 5.2 for such homotopies. The remaining steps in the proof, including the crossing of a critical level of $\rho$, require only inessential modifications which we leave to the reader.

Remarks. 1. It is possible to prove a stronger version of Theorem 4.1 with interpolation of a given holomorphic submersion along a closed complex subvariety $X_{0} \subset X$; compare with Theorem 2.5 in [F1].

2. Our proof also applies in the equidimensional case $\operatorname{dim} X=\operatorname{dim} Y=n$ as long as $Y$ satisfies Property $S_{n}$. Unfortunately we don't know any such example; the main case to be solved is $Y=\mathbb{C}^{n}$ (see Problems 1-3 in [F1]).

\section{Approximation of submersions on subsets of $\mathbb{C}^{n}$.}

In this section we prove Property $S_{n}$ and $H S_{n}$ for certain algebraic manifolds, in particular for those listed in Proposition 2.5.

A complex algebraic subvariety $A$ in an algebraic manifold $V$ will be called thin if it does not contain any complex hypersurfaces, i.e., $A$ has complex codimension at least two in $V$. The following is a key lemma.

LEMMA 5.1. Assume that $O \subset Q \subset \mathbb{C}^{n}$ are as in (2.1), (2.2). If $A$ is a thin algebraic subvariety of $\mathbb{C}^{n}$ with $A \cap O=\emptyset$ then any holomorphic submersion $h: \mathbb{C}^{n} \backslash A \rightarrow Y$ to a complex manifold $Y$ can be approximated uniformly on $O$ by holomorphic submersions $Q \rightarrow Y$ (and hence by submersions $\mathbb{C}^{n} \rightarrow Y$ ).

Proof. Let $z=\left(z^{\prime}, z^{\prime \prime}\right)$ be the coordinates on $\mathbb{C}^{n}$ with $z^{\prime}=\left(z_{1}, \ldots, z_{n-2}\right)$ and $z^{\prime \prime}=\left(z_{n-1}, z_{n}\right)$. After a small linear change of coordinates the projection $\pi: \mathbb{C}^{n} \rightarrow \mathbb{C}^{n-2}, \pi\left(z^{\prime}, z^{\prime \prime}\right)=z^{\prime}$, is proper when restricted to $A$. In this situation Lemma 3.4 from [F1] gives for any $\delta>0$ a holomorphic automorphism $\psi$ of $\mathbb{C}^{n}$ of the form $\psi\left(z^{\prime}, z^{\prime \prime}\right)=\left(z^{\prime}, \beta\left(z^{\prime}, z^{\prime \prime}\right)\right)$ satisfying $\sup _{z \in O}|\psi(z)-z|<\delta$ and 
$\psi(Q) \cap A=\emptyset$. Then $h \circ \psi: Q \rightarrow Y$ is a holomorphic submersion whose restriction to $O$ is uniformly close to $h$. This proves Lemma 5.1 .

Suppose now that $Y$ is a projective algebraic manifold of dimension $q$. Given a holomorphic submersion $f: O \rightarrow Y$ from a special convex set $O \subset \mathbb{C}^{n}$ for some $n>q$, our goal is to approximate $f$ uniformly on $O$ by a rational map $h: \mathbb{C}^{n} \rightarrow Y$ which is a holomorphic submersion outside of a thin algebraic subvariety $A \subset \mathbb{C}^{n}$ not intersecting $O$. If such approximations exist then by Lemma 5.1 we can approximate $h$ (and hence $f$ ) uniformly on $O$ by submersions $g: Q \rightarrow Y$, thus proving that $Y$ satisfies Property $S_{n}$. In a similar way we establish $H S_{n}$.

The possibility of approximating $f$ by $h$ is of course a nontrivial condition on $Y$ which fails for example on Kobayashi-Eisenman hyperbolic manifolds. We shall first establish Property $S_{n}$ of the projective spaces $\mathbb{C P}^{q}$ when $n>q$; the proof for Grassmanians and their Zariski open subsets with thin complements will follow the same pattern. (For $Y=\mathbb{C}^{q}$ see Proposition 3.3 in [F1].)

The quotient projection $\pi: \mathbb{C}_{*}^{q+1}=\mathbb{C}^{q+1} \backslash\{0\} \rightarrow \mathbb{C P}^{q}$ is a holomorphic fiber bundle with fiber $\mathbb{C}^{*}$, and by adding the zero section we obtain the universal line bundle $L \rightarrow \mathbb{C P}^{q}$. Assume that $f: O \rightarrow \mathbb{C P}^{q}$ is a holomorphic map on a compact convex set $O \subset \mathbb{C}^{n}$. Since $O$ is contractible, the bundle $f^{*} L \rightarrow O$ is topologically trivial and hence holomorphically trivial [O, G3]. Therefore $f^{*} L$ admits a nowhere vanishing holomorphic section which can be viewed as a holomorphic map $\tilde{f}: O \rightarrow \mathbb{C}_{*}^{q+1}$ satisfying $f=\pi \circ \widetilde{f}$ (a holomorphic lifting of $f$ ). We approximate $\tilde{f}$ uniformly on $O$ by a polynomial map $P: \mathbb{C}^{n} \rightarrow \mathbb{C}^{q+1}$ and take $h=\pi \circ P: \mathbb{C}^{n} \backslash P^{-1}(0) \rightarrow \mathbb{C P}^{q}$; by construction $\left.h\right|_{O}$ approximates $f$.

It remains to show that for a generic choice of $P$ the map $h$ is a submersion outside of a thin subvariety in $\mathbb{C}^{n}$. We say that $P$ is transverse to $\pi$ at a point $z \in \mathbb{C}^{n}$ if $P(z) \neq 0$ and $d P_{z}$ is transverse to the fiber of $\pi$ through $P(z)$. Note that $h=\pi \circ P$ is a submersion in a neighborhood of $z \in \mathbb{C}^{n}$ if and only if $P$ is transverse to $\pi$ at $z$. Since $f: O \rightarrow \mathbb{C P}^{q}$ is a submersion and hence $\tilde{f}$ is transverse to $\pi$ on $O$, we may assume (by choosing $P$ sufficiently uniformly close to $\tilde{f}$ on a neighborhood of $O$ ) that $P$ is also transverse to $\pi$ on $O$. To complete the proof it suffices to show that for a generic choice of $P$ the 'singularity set'

$$
\Sigma_{P}=P^{-1}(0) \cup\left\{z \in \mathbb{C}^{n} \backslash P^{-1}(0): d P_{z} \text { is not transverse to } \pi\right\}
$$

is a thin algebraic subset of $\mathbb{C}^{n}$ provided $n>q$.

Before proceeding we consider also the case when $Y=G_{k, m}$ is the Grassman manifold consisting of $k$-dimensional complex subspaces of $\mathbb{C}^{m}$. We apply the above proof with the fibration $\pi: V_{k, m} \rightarrow Y$ where $V_{k, m}$ is the Stiefel variety of all $k$-frames in $\mathbb{C}^{m}$ acted upon by the group $G L_{k}(\mathbb{C})$. We can identify $V_{k, m}$ with a Zariski open subset in $\mathbb{C}^{k m}$ with thin complement $B$ (which consists of $k \times m$ matrices with rank less than $k$ ), and $\pi$ defines an algebraic foliation $\mathcal{F}$ of $\mathbb{C}^{k m}$ which is nonsingular on $V_{k, m}$ and has homogeneous leaves biholomorphic 
to $G L_{k}(\mathbb{C})$. By Grauert's main theorem from [G3] we can lift any holomorphic map $f: O \rightarrow Y$ from a compact convex set $O \subset \mathbb{C}^{n}$ to a holomorphic map $\widetilde{f}: O \rightarrow V_{k, m}$ such that $f=\pi \circ \widetilde{f}$ (the argument is essentially the same as above). Next we approximate $\tilde{f}$ by a polynomial map $P: \mathbb{C}^{n} \rightarrow \mathbb{C}^{k m}$. We define the 'singularity set' $\Sigma_{P} \subset \mathbb{C}^{n}$ of $P$ as above, except that we replace the origin in the target space by $B=\mathcal{F}_{\text {sing }}$. To complete the proof it remains to show that, for $n>\operatorname{dim} Y$, the set $\Sigma_{P}$ is thin in $\mathbb{C}^{n}$ for a generic choice of $P$. The same proof applies to Zariski open sets $\Omega$ with thin complements in $G_{k, m}$; it suffices to add to $B$ the (thin) $\pi$-preimage of the complement of $\Omega$.

To complete the proof of Proposition 2.5 (at least the part concerning the Property $S_{n}$ ) we need the following. Fix a positive integer $N \in \mathbb{N}$ and denote by $V=\mathcal{P}(n, m, N)$ the vector space of all polynomial maps $P: \mathbb{C}^{n} \rightarrow \mathbb{C}^{m}$ of degree at most $N$; note that $V$ may be identified with a Euclidean space.

LEMMA 5.2. Let $\mathcal{F}$ be an algebraic foliation of codimension $q$ on $\mathbb{C}^{m}$ with thin singular locus $\mathcal{F}_{\text {sing }} \subset \mathbb{C}^{m}$. Given a polynomial map $P: \mathbb{C}^{n} \rightarrow \mathbb{\mathbb { C }}^{m}$ let $\Sigma_{P}$ consist of all points $z \in \mathbb{C}^{n}$ such that $P(z) \in \mathcal{F}_{\text {sing }}$ or $d P_{z}$ is not transverse to the leaf of $\mathcal{F}$ through $P(z)$. If $n>q$ then the set $\Sigma_{P}$ is thin for all $P$ outside of a proper algebraic subvariety of $\mathcal{P}(n, m, N)$.

The analogous result holds for any algebraic subsheaf $\mathcal{F}$ (not necessarily integrable) of the tangent sheaf of $\mathbb{C}^{m}$ for which $\mathcal{F}_{\text {sing }}$ (the locus of points where $\mathcal{F}$ is not locally free) is thin. The proof is a standard application of transversality arguments $[\mathrm{A}, \mathrm{Fo}]$ and is included only for completeness.

Proof of Lemma 5.2. Let $J \simeq \mathbb{\mathbb { C }}^{n+m+n m}$ denote the manifold of one-jets of holomorphic maps $\mathbb{C}^{n} \rightarrow \mathbb{C}^{m}$. For each holomorphic map $f: U \subset \mathbb{C}^{n} \rightarrow \mathbb{C}^{m}$ the associated one-jet extension is

$$
z \in U \rightarrow j_{z}^{1} f=\left(z, f(z), \partial_{1} f(z), \ldots, \partial_{n} f(z)\right) \in J
$$

where $\partial_{j}=\frac{\partial}{\partial \zeta_{j}}$. Denote by $p_{1}: J \rightarrow \mathbb{C}^{n}$ (resp. $p_{2}: J \rightarrow \mathbb{C}^{m}$ ) the source point (resp. the image point) projection.

Given an algebraic foliation $\mathcal{F}$ on $\mathbb{C}^{m}$ of codimension $q$ with singularity set $\mathcal{F}_{\text {sing }}$, let $\Sigma_{\mathcal{F}} \subset J$ denote the subset consisting of $p_{2}^{-1}\left(\mathcal{F}_{\text {sing }}\right)$ together with all one-jets over points $w \in \mathcal{F}_{\text {reg }}=\mathbb{C}^{m} \backslash \mathcal{F}_{\text {sing }}$ which are not transverse to $T_{w} \mathcal{F}$ (the tangent space to the leaf of $\mathcal{F}$ at $w$ ). We claim that $\Sigma_{\mathcal{F}}$ is a thin algebraic subset of $J$ provided that $\mathcal{F}_{\text {sing }}$ is thin and $n>q$. Clearly $p_{2}^{-1}\left(\mathcal{F}_{\text {sing }}\right)$ is thin. Furthermore, each point $w \in \mathcal{F}_{\text {reg }}$ is contained in a Zariski open set $U \subset \mathbb{C}^{m}$ such that the restricted foliation $\left.\mathcal{F}\right|_{U}$ is defined by one-forms $\omega_{1}, \ldots, \omega_{q}$ on $\mathbb{C}^{m}$ with polynomial coefficients which are pointwise independent at $w$. A one-jet $j_{z}^{1} f$ with $f(z)=w$ fails to be transverse to $T_{w} \mathcal{F}$ if and only if the $q \times n$ matrix with entries $\left\langle\omega_{j}(w), \partial_{k} f(z)\right\rangle(1 \leq j \leq q, 1 \leq k \leq n)$ has rank less than $q$. A simple count shows that the subvariety consisting of all such matrices has codimension $|n-q|+1$ which is $\geq 2$ when $n>q$. This gives over each point 
$w \in \mathcal{F}_{\text {reg }}$ at least two independent algebraic equations for $\Sigma_{\mathcal{F}}$, thus proving our claim.

We identify $V=\mathcal{P}(n, m, N)$ with the Euclidean space whose elements are collections $c=\left\{\left(c_{\alpha}\right):|\alpha| \leq N\right\}$, where $c_{\alpha} \in \mathbb{C}^{m}$ for each multiindex $\alpha=$ $\left(\alpha_{1}, \ldots, \alpha_{n}\right)$; the correspondence is given by

$$
c=\left\{c_{\alpha}:|\alpha| \leq N\right\} \longrightarrow P_{c}(z)=\sum_{|\alpha| \leq N} c_{\alpha} z^{\alpha} \in V .
$$

Let $\gamma: \mathbb{C}^{n} \times V \rightarrow J$ associate to every pair $(z, P)$ the one-jet $j_{z}^{1} P \in J$. Clearly $\gamma$ is polynomial in $z$ and linear in the coefficients of $P$. Furthermore, fixing $z \in \mathbb{C}^{n}$ and the coefficients $c_{\alpha}$ with $2 \leq|\alpha| \leq N, \gamma$ gives a linear map of maximal rank (a linear submersion) from the space of coefficients $\left\{c_{\alpha}:|\alpha| \leq 1\right\}$ of order $\leq 1$ onto the fiber $p_{1}^{-1}(z) \subset J$. It follows that $\Sigma^{1}:=\gamma^{-1}\left(\Sigma_{\mathcal{F}}\right)$ is a thin algebraic subvariety of $\mathbb{C}^{n} \times V$. Hence for every $P$ outside a proper algebraic subvariety of $V$ the set $\Sigma_{P}=\left\{z \in \mathbb{C}^{n}:(z, P) \in \Sigma^{1}\right\}$ is a thin subvariety of $\mathbb{C}^{n}$. By construction, $\Sigma_{P}$ is the set of point $z \in \mathbb{C}^{n}$ at which $d P_{z}$ fails to be transverse to the foliation $\mathcal{F}$ (or $P(z)$ belongs to $\mathcal{F}_{\text {sing }}$ ). This proves Lemma 5.2 .

This establishes Property $S_{n}$ of any complex manifold $Y$ in Proposition 2.5 for all $n>\operatorname{dim} Y$. The proof that $\mathbb{C P}^{1}$ also satisfies Property $S_{1}$ requires a slightly different argument as follows. Let $f: O \rightarrow \mathbb{C P}^{1}=\mathbb{C} \cup\{\infty\}$ be a holomorphic submersion from a convex set $O \subset \mathbb{C}$ to the Riemann sphere. Then $f^{-1}(\infty) \subset O$ is a finite subset of $O$ which we may assume to be contained in the interior of $O$. Let $B \subset \mathbb{C}$ be a convex set such that $B \cap f^{-1}(\infty)=\emptyset$, $B \cup O$ is also convex, and $\overline{B \backslash O} \cap \overline{O \backslash B}=\emptyset$. Let $C=B \cap O$. By Theorem 3.1 in $[\mathrm{F} 1]$ we can approximate $\left.f\right|_{C}: C \rightarrow \mathbb{C}=\mathbb{C P}^{1} \backslash\{\infty\}$ by entire noncritical functions $g: \mathbb{C} \rightarrow \mathbb{C}$. Proceeding as in the proof of Proposition 4.2 above we find a biholomorphic transition map $\gamma$ between $f$ and $g$ on a neighborhood of $C$, decompose $\gamma=\beta \circ \alpha^{-1}$ and thus patch $f$ and $g$ into a submersion $\widetilde{f}: B \cup O \rightarrow \mathbb{C P}^{1}$ which approximates $f$ on $O$. Furthermore we can arrange that $\widetilde{f}^{-1}(\infty)=f^{-1}(\infty)$. In finitely many steps of this kind we approximate $f$ by a submersion $Q \rightarrow \mathbb{C P}^{1}$ as desired.

We now show that every manifold $Y$ as above also satisfies Property $H S_{n}$ for all $n>q=\operatorname{dim} Y$. Recall that we have a submersion $\pi$ : $\mathbb{C}^{m} \backslash B \rightarrow Y$ onto $Y$ where $B$ is thin in $\mathbb{C}^{m}$ and contains $\mathcal{F}_{\text {sing }}$. Let $O \subset Q \subset \mathbb{C}^{n}$ be as in (2.1), (2.2). Assume that $f_{t}: O \subset \mathbb{C}^{n} \rightarrow Y(t \in[0,1])$ is a homotopy of holomorphic submersions such that $f_{0}$ and $f_{1}$ extend to submersions $Q \rightarrow Y$. By the same argument as in the proof of Property $S_{n}$ we can lift $\left\{f_{t}\right\}$ to a homotopy of maps $\widetilde{f}_{t}: O \rightarrow \mathbb{C}^{m}(t \in[0,1])$ which are transverse to the foliation $\mathcal{F}$ defined by $\pi$ and such that $\widetilde{f}_{0}, \widetilde{f}_{1}$ are defined and transverse to $\mathcal{F}$ on $Q$. We approximate $\left\{\widetilde{f}_{t}\right\}$ by a homotopy of polynomial maps $P_{t}: \mathbb{C}^{n} \rightarrow \mathbb{C}^{m}$ which also depends polynomially on a parameter $t \in \mathbb{C}$ (the approximation of $\widetilde{f}_{t}$ by $P_{t}$ takes place on $O$ for $t \in(0,1)$ and on $Q$ for $t=0$ and $t=1)$. 
Let $N$ be the maximal degree of the maps $P_{t}$ in the above family, considered as polynomials both in $t \in \mathbb{C}$ and $z \in \mathbb{C}^{n}$. Denote by $\widetilde{\mathcal{P}}(n, m, N)$ the space of all polynomial maps $(t, z) \in \mathbb{C} \times \mathbb{C}^{n} \rightarrow \mathbb{C}^{m}$ of degree $\leq N$. For every $P \in$ $\widetilde{\mathcal{P}}(n, m, N)$ and $t \in \mathbb{C}$ we have $P_{t}=P(t, \cdot) \in \mathcal{P}(n, m, N)$. The proof of Lemma 5.2 shows that for a generic choice of $P \in \widetilde{\mathcal{P}}(n, m, N)$ the 'singularity set' $\Sigma_{P} \subset \mathbb{C}^{1+n}$, consisting of all $(t, z)$ for which the one-jet $j_{z}^{1} P_{t}$ is not transverse to $\mathcal{F}$ (or $P_{t}(z) \in B$ ), is a thin algebraic subvariety of $\mathbb{\mathbb { C }}^{1+n}$. Hence for all but finitely many $t \in \mathbb{C}$ the singularity set $\Sigma_{t} \subset \mathbb{C}^{n}$ of the map $P_{t}$ is also thin. By a small deformation of the segment $[0,1] \subset \mathbb{R}$ inside $\mathbb{C}$ we may can avoid this finite exceptional set of $t$ 's, thus obtaining a homotopy $P_{t}: \mathbb{C}^{n} \rightarrow \mathbb{C}^{m}$ of polynomial maps approximating $\widetilde{f}_{t}(t \in[0,1])$ such that $\Sigma_{t}$ is thin for all $t \in[0,1]$. Hence $h_{t}=\pi \circ P_{t}: \mathbb{C}^{n} \backslash \Sigma_{t} \rightarrow Y$ is a homotopy of submersions which approximates the original homotopy $f_{t}: O \rightarrow Y$ uniformly on $O$, and uniformly with respect to the parameter $t$.

We can now conclude the proof as in Lemma 5.1: Applying [F1, Lemma 3.4] with the additional parameter $t \in[0,1]$ we obtain a family of holomorphic automorphisms $\psi_{t}\left(z^{\prime}, z^{\prime \prime}\right)=\left(z^{\prime}, \beta_{t}\left(z^{\prime}, z^{\prime \prime}\right)\right)$ of $\mathbb{C}^{n}$, depending smoothly on $t \in$ $[0,1]$, such that for every $t$ we have $\psi_{t}(Q) \cap \Sigma_{t}=\emptyset,\left.\psi_{t}\right|_{O}$ is close to the identity map on $O$, and the maps $\psi_{0}$ and $\psi_{1}$ are close to the identity map on $Q$. The homotopy of holomorphic submersions $h_{t} \circ \psi_{t}: Q \rightarrow Y$ satisfy all the required properties, except perhaps the interpolation condition at the endpoints $t=0,1$ which is easily fixed.

When proving the Property $S_{n}$ or $H S_{n}$ for $\mathbb{C}^{q}$ (or a Zariski open subset with thin complement in $\mathbb{C}^{q}$ ) we proceed as above but skip the first step, i.e, we can directly deal with polynomial maps $\mathbb{C}^{n} \rightarrow \mathbb{C}^{q}$. This completes the proof of Proposition 2.5.

Remark. Our proof of Property $H S_{n}$ breaks down for multi-parameter families of submersions: we still prove that for a generic choice of the polynomial map $P: \mathbb{C}^{k} \times \mathbb{\mathbb { C }}^{n} \rightarrow \mathbb{C}^{m}$ its singularity set $\Sigma_{P} \subset \mathbb{C}_{t}^{k} \times \mathbb{\mathbb { C }}_{z}^{n}$ (with $t \in \mathbb{\mathbb { C }}^{k}$ being the parameter) is thin; hence for all $t$ outside of a proper algebraic subvariety $Z \subset \mathbb{C}^{k}$ the singularity set $\Sigma_{t} \subset \mathbb{C}^{n}$ of $P_{t}=P(t, \cdot): \mathbb{C}^{n} \rightarrow \mathbb{C}^{m}$ is thin in $\mathbb{C}^{n}$. However, when $k>1$ we may not be able to avoid the exceptional set $Z$ by a small deformation of the parameter cube $[0,1]^{k} \subset \mathbb{R}^{k}$ in $\mathbb{C}^{k}$. Such a multiparameter analogue of Property $H S_{n}$ would be needed to obtain the complete parametric homotopy principle for submersions $X \rightarrow Y$.

\section{A simple proof of the Oka-Grauert-Gromov theorem.}

We give a simple proof of the following result from [F3].

THEOREM 6.1. Let $h: Z \rightarrow X$ be a holomorphic fiber bundle over a Stein manifold $X$. If the fiber $Y=h^{-1}(x)(x \in X)$ admits a finite dominating collection of sprays then the inclusion $\operatorname{Holo}(X, Z) \hookrightarrow \operatorname{Cont}(X, Z)$ of the space of holomorphic sections into the space of continuous sections is a weak homotopy equivalence. 
The spaces of sections are equipped with the compact-open topology. For the definition of 'dominating families of sprays' see [F3]. The classical case when $Z$ is a principal holomorphic bundle (with fiber a complex Lie group or homogeneous space) is due to Grauert [G3]. The case when the fiber $Y$ admits a dominating spray is due to Gromov [Gr4, Sec. 2.8], and a detailed proof can be found in [FP1].

Proof. We use the scheme of proof of Theorem 2.1 in Sect. 4 (which is similar to the one in [FP1]). We may assume that the fiber $Y$ is connected.

At a noncriticial step we have a continuous section $f: X \rightarrow Z$ which is holomorphic in an open neighborhood of a smoothly bounded, compact, strongly pseudoconvex domain $A \subset X$. We attach a small 'convex bump' $B$ satisfying

(i) $\overline{A \backslash B} \cap \overline{B \backslash A}=\emptyset$ and the union $A \cup B$ is again smoothly bounded strongly pseudoconvex,

(ii) in some local holomorphic coordinates in a neighborhood $U \subset X$ of $B$ the sets $B$ and $C=A \cap B$ are compact convex subset of $\mathbb{C}^{n}(n=\operatorname{dim} X)$, and

(iii) the restricted bundle $\left.Z\right|_{U}$ is trivial, $\left.Z\right|_{U} \simeq U \times Y$.

The set $B$ can be a 'special convex set' of the form (2.2) in some local coordinates on $X$. The goal is to approximate $f$ uniformly on $A$ as close as desired by a continuous section $\widetilde{f}: X \rightarrow Z$ which is holomorphic in a neighborhood of $A \cup B$. This is accomplished by the 'noncritical case' in [Gr4] or [FP1, Sect. 6] and here we do not propose any changes. We recall the main steps for the sake of the reader.

Let $f_{1}:\left.U \rightarrow Z\right|_{U} \simeq U \times Y$ be any holomorphic section (for instance, a constant section). Since $C$ is convex and hence holomorphically contractible, there is a homotopy $\left\{f_{t}\right\}_{t \in[0,1]}$ of holomorphic sections over a neighborhood $V \subset U$ of $C$ connecting $f_{0}:=\left.f\right|_{V}$ to $\left.f_{1}\right|_{V}$. Since $Y$ admits a finite dominating family of sprays, the homotopy version of the Oka-Weil theorem [F3, Theorem 3.1] gives a uniform approximation of the homotopy $\left\{f_{t}\right\}$ on a smaller neighborhood of $C$ by a homotopy of sections $\left\{\widetilde{f}_{t}\right\}_{t \in[0,1]}$ which are holomorphic over a neighborhood of $B$, with $\widetilde{f}_{1}=f_{1}$ and $g:=\widetilde{f}_{0}$ very close to $f$ on a neighborhood of $C$. (This approximation result is essentially due to Grauert [G1, G2]; see also [Gr4] and [FP1].) Applying Theorem 4.1 in [F3] (or Theorem 5.1 in [FP1] when $Y$ admits a dominating spray) we glue the pair of sections $f, g$ into a section $\tilde{f}$ which is holomorphic in an open neighborhood of $A \cup B$ and extends to a continuous section over $X$. This completes the noncritical step.

A complication in this process arises when crossing a critical level $\left\{\rho=c_{0}\right\}$ of the given strongly plurisubharmonic exhaustion function $\rho: X \rightarrow \mathbb{R}$. In that case we have $A=\{\rho \leq c\}$ for some $c<c_{0}$ close to $c_{0}$ (such that $\rho$ has no critical values on $\left.\left[c, c_{0}\right)\right)$. The set $B$ is a 'handle' attached to $A$ such that the attaching set $C=A \cap B$ is no longer contractible. (The union $A \cup B$ is diffeomorphic to the sublevel set $\left\{\rho \leq c^{\prime}\right\}$ for some $c^{\prime}>c_{0}$.) In this case we cannot find the desired homotopy $\left\{f_{t}\right\}$ as above. In [FP1] the difficulty 
was avoided by applying the noncritical case with an additional parameter to construct $\left\{f_{t}\right\}$, beginning at the 'core' of $C$ (which is a totally real torus) and performing 'approximation and gluing' until reaching $C$ in finitely many steps (Theorem 4.5 in [FP1]). An alternative method, proposed by Gromov [Gr4] and developed in [FP2], uses a more complicated induction scheme and remains applicable even if the submersion $Z \rightarrow X$ is not locally trivial, as long it admits fiber-dominating sprays over small open subsets of $X$.

Here we propose a simple alternative way to pass the critical level of $\rho$ by applying Theorem 3.2. Assume that $A$ and $f$ are as above. We attach to $A$ a smooth totally real handle $M$ passing through the critical point $p$, with $\operatorname{dim} M$ equal the Morse index of $p$ (see Sect. 4, Step 1: extension to a handle). By Theorem 3.2 we can approximate $f$ uniformly on $A \cup M$ by a section $\widetilde{f}$ which is holomorphic in a neighborhood of $A \cup M$ and continuous on $X$ (compare with Step 2 in Sect. 4). The proof is completed by Step 3 in Sect. 4 without any changes (i.e., we use the noncritical case with a different strongly plurisubharmonic function $\tau$ in order to pass the critical level of $\rho$ at $p$, then we revert back to $\rho$ and proceed by the noncritical case to the next critical level of $\rho$.) All steps adapt easily to the parametric case and hence we obtain the full statement of Theorem 1.4 in [FP1] under the weaker assumption that the fiber admits a dominating family of sprays (instead of a dominating spray). This completes the proof.

Comparing with the proof of Theorem 2.1 we see that the only essential difference lies in the method of local approximation and gluing of pairs of sections. In the proof of Theorem 6.1 we use a dominating family of sprays on the fiber to linearize the problem. On the other hand, in Theorem 2.1 we patch a pair of submersions by decomposing the transition map between them in the source manifold $X$ without using any properties of the target manifold (these are only used for the approximation). The method of passing a critical level is identical in both proofs, except that the construction of submersions requires a maximal rank extension of the one-jet across the handle.

The above may be conceptually the simplest available proof of the OkaGrauert-Gromov principle, even in Grauert's classical case concerning principal bundles with homogeneous fibers. Unlike in the earlier papers, holomorphic sections are constructed without using the intermediate technical devices for multi-parameter homotopies of sections.

Acknowledgements. This research has been supported in part by a grant from the Ministry of Science and Education of the Republic of Slovenia. Kind thanks to J. Winkelmann for helpful discussions regarding Property $S_{n}$.

\section{References}

[A] ABRAHAM, R., Transversality in manifolds of mappings. Bull. Amer. Math. Soc., 69 (1963), 470-474. 
[B] BARTH, W., PETERS, C., VAN DE VEN, A., Compact Complex Surfaces. Springer, Berlin-Heidelberg-New Zork-Tokyo, 1984.

[De] DEMAILLY, J.-P., Cohomology of $q$-convex spaces in top degrees. Math. Z., 204 (1990), 283-295.

[DG] DOCQUIER, F., GRAUERT, H., Levisches Problem und Rungescher Satz für Teilgebiete Steinscher Mannigfaltigkeiten. (German) Math. Ann., 140 (1960), 94-123.

[E] ELIASHBERG, Y., Topological characterization of Stein manifolds of dimension > 2. Internat. J. Math., 1 (1990), 29-46.

[EM] ELIASHBERG, Y., MISHACHEV, N., Introduction to the h-principle. Graduate Studies in Math., 48. Amer. Math. Soc., Providence, RI, 2002.

[Fo] FORSTER, O., Plongements des variétés de Stein. Comment. Math. Helv., 45 (1970), 170-184.

[F1] FORSTNERIČ, F., Noncritical holomorphic functions on Stein manifolds. Acta Math., 191 (2003). [arXiv: math.CV/0211112]

[F2] — , The homotopy principle in complex analysis: A survey, in Explorations in Complex and Riemannian Geometry: A Volume dedicated to Robert E. Greene (J. Bland, K.-T. Kim, and S. G. Krantz, eds., 7399). Contemporary Mathematics, 332, American Mathematical Society, Providence, 2003.

[F3] - The Oka principle for sections of subelliptic submersions. Math. Z., 241 (2002), 527-551.

[FK] FORSTNERIČ, F., KOZAK, J., Strongly pseudoconvex handlebodies. J. Korean Math. Soc., 40 (2003), 727-746.

[FLØ] FORSTNERIČ, F., LØW, E., ØVRELID, N., Solving the $d$ - and $\bar{\partial}$-equations in thin tubes and applications to mappings. Michigan Math. J., 49 (2001), 369-416.

[FP1] FORSTNERIČ, F., PREZELJ, J., Oka's principle for holomorphic fiber bundles with sprays. Math. Ann., 317 (2000), 117-154.

[FP2] - Oka's principle for holomorphic submersions with sprays. Math. Ann., 322 (2002), 633-666.

[FP3] - Extending holomorphic sections from complex subvarieties. Math. Z., 236 (2001), 43-68.

[FR] FORSTNERIČ, F., ROSAY, J.-P., Approximation of biholomorphic mappings by automorphisms of $\mathbb{C}^{n}$. Invent. Math., 112 (1993), 323-349. Erratum, Invent. Math., 118 (1994), 573-574.

[G1] GRAUERT, H., Approximationssätze für holomorphe Funktionen mit Werten in komplexen Räumen. Math. Ann., 133 (1957), 139-159.

[G2] - Holomorphe Funktionen mit Werten in komplexen Lieschen Gruppen. Math. Ann., 133 (1957), 450-472. 
[G3] — Analytische Faserungen über holomorph-vollständigen Räumen. Math. Ann., 135 (1958), 263-273.

[Gr1] GROMOV, M., Stable maps of foliations into manifolds. Izv. Akad. Nauk, S.S.S.R., 33 (1969), 707-734.

[Gr2] - Convex integration of differential relations, I. (Russian) Izv. Akad. Nauk SSSR Ser. Mat., 37 (1973), 329-343. English translation in Math. USSR-Izv., 7 (1973), 329-343.

[Gr3] — , Partial Differential Relations. Ergebnisse der Mathematik und ihrer Grenzgebiete (3), 9. Springer, Berlin-New York, 1986.

[Gr4] — , Oka's principle for holomorphic sections of elliptic bundles. $J$. Amer. Math. Soc., 2 (1989), 851-897.

[GN] GUNNING, R. C., NARASIMHAN, R., Immersion of open Riemann surfaces. Math. Ann., 174 (1967), 103-108.

[GR] GUNNING, R. C., ROSSI, H., Analytic functions of several complex variables. Prentice-Hall, Englewood Cliffs, 1965.

[HL1] HENKIN, G. M., LEITERER, J., Andreotti-Grauert Theory by Integral Formulas. Progress in Math., 74, Birkhäuser, Boston, 1988.

[HL2] — , The Oka-Grauert principle without induction over the basis dimension. Math. Ann., 311 (1998), 71-93.

[Hö1] HÖRMANDER, L., $L^{2}$ estimates and existence theorems for the $\bar{\partial}$ operator. Acta Math., 113 (1965), 89-152.

[Hö2] — An Introduction to Complex Analysis in Several Variables. Third ed. North Holland, Amsterdam, 1990.

[HW] HÖRMANDER, L., WERMER, J., Uniform approximations on compact sets in $\mathbb{C}^{n}$. Math. Scand., 23 (1968), 5-21.

[O] OKA, K., Sur les fonctions des plusieurs variables. III: Deuxième problème de Cousin. J. Sc. Hiroshima Univ., 9 (1939), 7-19.

[P] PHILLIPS, A., Submersions of open manifolds. Topology, 6 (1967), 170206.

[RS] RANGE, R. M., SIU, Y. T., $\mathcal{C}^{k}$ approximation by holomorphic functions and $\bar{\partial}$-closed forms on $\mathcal{C}^{k}$ submanifolds of a complex manifold. Math. Ann., 210 (1974), 105-122.

[Ro] ROSAY, J.-P., A counterexample related to Hartog's phenomenon (a question by E. Chirka). Michigan Math. J., 45 (1998), 529-535.

[S] SIU, J.-T., Every Stein subvariety admits a Stein neighborhood. Invent. Math., 38 (1976), 89-100.

[W] WINKELMANN, J., The Oka-principle for mappings between Riemann surfaces. Enseign. Math. (2) 39 (1993), 143-151.

Address: Institute of Mathematics, Physics and Mechanics, University of Ljubljana, Jadranska 19, 1000 Ljubljana, Slovenia 
E-mail: franc.forstneric@fmf.uni-lj.si 